\title{
Emission-lines calibrations of the star formation rate from the Sloan Digital Sky Survey
}

\author{
B. Argence ${ }^{1,2}$ and F. Lamareille ${ }^{1,3}$ \\ ${ }^{1}$ Laboratoire d'Astrophysique de Toulouse-Tarbes, Université de Toulouse, CNRS, 14 avenue Édouard Belin, \\ 31400 Toulouse, France \\ e-mail: argence@apc.univ-paris7.fr \\ 2 APC, UMR 7164, CNRS, Université Paris 7 Denis Diderot, 10 rue Alice Domon et Léonie Duquet, 75025 Paris Cedex 13, France \\ 3 Osservatorio Astronomico di Bologna, via Ranzani 1, 40127 Bologna, Italy \\ e-mail: flamare@ast.obs-mip.fr
}

Received 21 December 2006 / Accepted 6 December 2008

ABSTRACT

\begin{abstract}
Aims. Up to now, the study of the star formation rate in galaxies has been based mainly on the $\mathrm{H} \alpha$ emission-line luminosity. However, this standard calibration cannot be applied at all redshifts if there is only one instrumental setup. Surveys based on optical spectroscopy do not observe the $\mathrm{H} \alpha$ emission line at redshifts higher than $z \sim 0.5$. Our goal is to study existing star formation rate calibrations and to provide new ones, still based on emission-line luminosities, which can be applied for various instrumental setups.

Methods. We used the SDSS public data release DR4, which gives star formation rates and emission-line luminosities for more than 100000 starforming galaxies observed at low redshifts. We take advantage of this statistically significant sample in order to study the relations, based on these data, between the star formation rate and the luminosities of some well-chosen emission lines. We correct the emission-line measurements for dust attenuation using the same attenuation curve as the one used to derive the star formation rates. Results. We confirm that the best results are obtained when relating star formation rates to the $\mathrm{H} \alpha$ emission-line luminosity, itself corrected for dust attenuation. This calibration has an uncertainty of 0.17 dex. We show that one has to carefully check the method used to derive the dust attenuation and to use the adequate calibration: in some cases, the standard scaling law has to be replaced by a more general power law. When data are corrected for dust attenuation but the $\mathrm{H} \alpha$ emission line not observed, the use of the $\mathrm{H} \beta$ emission line, if possible, has to be preferred to the [OII] $\lambda 3727$ emission line. In the case of uncorrected data, the correction for dust attenuation can be assumed as a constant mean value, but we show that such a method leads to poor results in terms of dispersion and residual slope. Self-consistent corrections, such as previous studies based on the absolute magnitude, give better results in terms of dispersion but still suffer from systematic shifts and/or residual slopes. The best results with data not corrected for dust attenuation are obtained when using the observed [OII] $\lambda 3727$ and $\mathrm{H} \beta$ emission lines together. This calibration has an uncertainty of 0.23 dex.
\end{abstract}

Key words. galaxies: fundamental parameters - galaxies: statistics - ISM: HII regions - ISM: dust, extinction

\section{Introduction}

Massive ongoing spectroscopic surveys of the high-redshift Universe allow us to draw a new picture of how the galaxies evolve through cosmic times. Many physical properties are used as tracers of the evolutionary stage and the past history of one galaxy (e.g. stellar mass, metallicity, amount of dust, star formation rate). Thanks to extensive works on stellar population models (Bruzual \& Charlot 2003; Fioc \& Rocca-Volmerange 1997) and photo-ionization models (Charlot \& Longhetti 2001), most of these parameters can now be recovered precisely from rest-frame optical spectroscopic observations, using all the information available from the stellar continuum and absorptionor emission-line measurements.

Unfortunately, these models often require a better signalto-noise ratio, wavelength coverage, and/or spectral resolution than the ones available in recent deep surveys (e.g. VVDS: Le Fèvre et al. 2005; zCOSMOS: Lily et al. 2007). Thus, a simpler approach is to instead use emission-line diagnostics that would be calibrated on high-quality samples, taken from local Universe surveys (e.g. 2dFGRS: Colless et al. 2001; SDSS: York et al. 2000). This approach has already been extensively used with older data to recover metallicities with oxygen-to-hydrogen (McGaugh 1991; Kewley \& Dopita 2002) or nitrogen-to-hydrogen (Pettini \& Pagel 2004; van Zee et al. 1998) line ratios, dust content with the $\mathrm{H} \alpha / \mathrm{H} \beta$ Balmer decrement (Seaton 1979; Calzetti 2001), or star formation rates with the $\mathrm{H} \alpha$ or [OII] $\lambda 3727$ emission-line luminosities (Kennicutt 1998; Kewley et al. 2004).

Recently, the star formation rates of $>100000$ SDSS starforming galaxies have been estimated using the CL01 method (Charlot \& Longhetti 2001), which takes all information into account that is available in emission-line measurements (Brinchmann et al. 2004). Then, the emission-line measurements of the same galaxies have also been made publicly available. We can thus use this large amount of data to derive new goodquality calibrations of the star formation rates (hereafter SFR) from emission-line luminosities.

The standard calibration of the SFR versus the $\mathrm{H} \alpha$ luminosity is based on a very simple scaling relation, driven by the amount of $\mathrm{H} \alpha$ recombination photons, which are directly proportional to the intensity of the ionizing source, i.e. the amount of young, hot stars, which the SFR is an indicator of (Kennicutt 1998). The only drawback to this simple assumption is that the $\mathrm{H} \alpha$ luminosity has to be corrected for dust attenuation. 
As shown by Kewley et al. (2004), the calibration of the SFR versus the [OII] $\lambda 3727$ luminosity is not only more affected by dust attenuation, but it also depends on metallicity, since interstellar medium with higher gas-phase metallicities produces fewer [OII] $\lambda 3727$ photons because of increased oxygen cooling. Starting from this result, they have derived new calibrations of the SFR versus [OII] 33727 luminosity, which take the metallicity into account. The idea was to calibrate the [OII] $\lambda 3727 / \mathrm{H} \alpha$ emission-line ratio versus the metallicity, and then to use it to recover the SFR from the $\mathrm{H} \alpha$ luminosity, itself derived from the $[\mathrm{OII}] \lambda 3727$ luminosity. The dependence on the metallicity of the $[\mathrm{OII}] \lambda 3727 / \mathrm{H} \alpha$ emission-line ratio, or more generally the [OII] $\lambda 3727$ based SFR calibrations, has been later confirmed by Mouhcine et al. (2005) and Bicker \& Fritze-v. Alvensleben (2005).

Recently, Moustakas et al. (2006) and Weiner et al. (2007) have derived a new calibration of the SFR of galaxies versus [OII] $\lambda 3727$ luminosity. They deal with the effect of the metallicity with a slightly different approach, by parametrizing their calibration in terms of the $B$-band luminosity, which can be used as a rough metallicity indicator because of the metallicityluminosity relation (Lequeux et al. 1979; Skillman et al. 1989; Richer \& McCall 1995; Lamareille et al. 2004; Tremonti et al. 2004; Lamareille et al. 2006).

We point out that these previous studies base their new calibrations on either a direct estimate or a secondary indicator (e.g. rest-frame luminosity) of the metallicity, which in both cases suffers from intrinsic uncertainties and degeneracies (see Kewley \& Ellison 2008, for a detailed discussion of the uncertainties of metallicity calibrations, or Lamareille et al. 2004, for a discussion of the dispersion of the luminosity-metallicity relation). Moreover, some of these previous works also assume that the dust attenuation is known, whereas it is difficult to estimate this quantity when the $\mathrm{H} \alpha$ emission line is not measured (which is the case when one wants to use [OII] $\lambda 3727$ instead to derive SFR).

The paper is organized as follows. We first present the sample we used and the selection we applied to it (Sect. 2), and then we calibrate the SFR as a function of emission-line luminosities when a correction for dust attenuation is available (Sect. 3), or when it is not available (Sect. 4). We finally draw some conclusions (Sect. 5).

\section{Description of the sample}

The physical properties of a total of 567486 galaxy spectra inside SDSS Data Release 4 (DR4, Adelman-McCarthy et al. 2006) have been made publicy available on the following website: http : //www . mpa-garching . mpg . de/SDSS/. Taking advantage of this huge amount of data, we made a subcatalog with the SFR, the emission-line measurements, the spectral classification, and the metallicities of a subsample of galaxies. The selection will be described later in this section after we first describe, for the benefit of the reader, the main ingredients of the methods used to derive the physical parameters.

\subsection{Physical properties}

The spectral properties of SDSS galaxies have been measured with "platefit" software by Tremonti et al. (2004). For each spectrum, they carefully subtract the stellar continuum and absorption lines by fitting a linear combination of single stellar population models of different ages (Bruzual \& Charlot 2003). Then they fit all emission lines at the same time as a unique nebular spectrum in which each line is modeled by a Gaussian.
Thanks to subtracting of the stellar component, Balmer emission lines are automatically corrected for underlying absorption. The [OII] $\lambda 3727$ line that we use in this study is the sum of two Gaussians of the same width modeling the [OII] $\lambda \lambda 3726,3729$ doublet. The AGN classification is based on the $[\mathrm{OIII}] \lambda 5007 / \mathrm{H} \beta$ vs. [NII] $\lambda 6584 / \mathrm{H} \alpha$ emission-line diagnostic diagram and has been performed by Kauffmann et al. (2003a).

The SFR and gas-phase oxygen abundances were computed from emission-lines fluxes using the Charlot \& Longhetti (2001, hereafter CL01) method by Brinchmann et al. (2004) and Tremonti et al. (2004), respectively. This method compares all emission-lines fluxes together to a set of theoretical nebular spectra, which are modeled with five parameters: the metallicity, the ionization degree, the dust-to-metal ratio, the dust attenuation, and the SFR efficiency factor. For each galaxy, one estimate of each observed parameter is computed with a Bayesian approach (see Brinchmann et al. 2004, for more details). We note that the SFRs are computed assuming the Kroupa (2001) initial mass function (IMF). One may scale the SFR estimates discussed in this paper to the Salpeter (1955) IMF by multiplying them by a factor 1.5 (or by adding 0.176 dex to their logarithm) and to the Chabrier (2003) IMF by multiplying them by a factor 0.88 (or by subtracting 0.056 dex to their logarithm). The CL01 SFR will be used as the reference SFR throughout this study.

We computed the luminosity $L$ of each line starting from its measured flux $F$, and the redshift $z$ of the galaxy, using the equation

$$
\begin{aligned}
& L=4 \pi\left(\frac{c}{H_{0}} \cdot(1+z) \cdot \int_{0}^{z} f\left(z^{\prime}\right)^{-1 / 2} \mathrm{~d} z^{\prime}\right)^{2} \times F \\
& f\left(z^{\prime}\right)=\left(1+z^{\prime}\right)^{2}\left(1+\Omega_{\mathrm{m}} z^{\prime}\right)-\Omega_{\Lambda} z^{\prime}\left(2+z^{\prime}\right) .
\end{aligned}
$$

The luminosities were calculated with the same cosmology based on WMAP results (Spergel et al. 2003) as the one used by Brinchmann et al. (2004) to estimate the SFR: $H_{0}=$ $70 \mathrm{~km} \mathrm{~s}^{-1} \mathrm{Mpc}^{-1}, \Omega_{\Lambda}=0.7$, and $\Omega_{\mathrm{m}}=0.3$. All the calibrations provided in this study are independent of the cosmology. They indeed compare SFR and emission-line luminosities which are affected in the same way by the distance modulus.

Finally, we have cross-matched this catalog with the VAGC catalog (Blanton et al. 2005) to get the $k$-corrected absolute magnitudes which are used in this study. The absolute magnitude in the $B$-band is derived from SDSS $u^{\prime}$ - and $g^{\prime}$-bands using Eq. (1) of Moustakas et al. (2006).

\subsection{Dust attenuation}

We use the following notations in this work: the observed flux of the three emission lines used in this study are designated by the name of the line: $\mathrm{H} \alpha, \mathrm{H} \beta$, and [OII] (stands for [OII] $\lambda 3727$ ). The intrinsic flux corrected for dust attenuation is designated by a superscript $\mathrm{i}: \mathrm{H} \alpha^{\mathrm{i}}, \mathrm{H} \beta^{\mathrm{i}}$, and [OII $]^{\mathrm{i}}$. The attenuation law is designed by the function $\tau(\lambda)$, and the effective dust attenuation in $V$-band by the symbol $\tau_{V}$. For simplification purposes, we also note: $\tau(V)=\tau(5500 \AA)(V$-band $), \tau(\beta)=\tau(4861 \AA)$, $\tau(\alpha)=\tau(6563 \AA)$, and $\tau(\mathrm{OII})=\tau(3727 \AA)$. The intrinsic flux $f_{\lambda}^{\mathrm{i}}(\lambda)$ at any wavelength $\lambda$ as a function of the observed flux $f_{\lambda}(\lambda)$ is given by the following equation:

$f_{\lambda}^{\mathrm{i}}(\lambda)=f_{\lambda}(\lambda) \cdot \exp \left(\frac{\tau_{V}}{\tau(V)} \cdot \tau(\lambda)\right)$. 
We computed the dust attenuation by comparing the observed to the intrinsic $\mathrm{H} \alpha / \mathrm{H} \beta$ emission-line ratios, using the following equation:

$\tau_{V}^{\mathrm{Balmer}}=\frac{\ln (\mathrm{H} \alpha / \mathrm{H} \beta)-\ln \left(\mathrm{H} \alpha^{\mathrm{i}} / \mathrm{H} \beta^{\mathrm{i}}\right)}{\tau(\beta)-\tau(\alpha)} \cdot \tau(V)$

In the following work, we use the standard case $\mathrm{B}$ recombination ratio (hereafter standard intrinsic Balmer ratio): $\mathrm{H} \alpha^{\mathrm{i}} / \mathrm{H} \beta^{\mathrm{i}}=2.85$ (Osterbrock 1989) and the Charlot \& Fall (2000) mean attenuation curve: $\tau(\lambda) \propto \lambda^{-0.7}$. The choice of this curve is made to be consistent with the setup used to compute the SFRs in the SDSS DR4 data (the SFR is computed self-consistently with correction for dust attenuation).

The dust attenuation recovered with an assumed constant intrinsic Balmer ratio $\left(\tau_{V}^{\text {Balmer }}\right)$ does not take the variations in this ratio into account with other physical parameters (e.g. metallicity). We thus also use the true dust attenuation recovered with CL01 models by Brinchmann et al. (2004). We designate it by the symbol $\tau_{V}^{\mathrm{CL} 01}$. The intrinsic flux corrected for dust attenuation using this parameter is designated by a superscript $i C: \mathrm{H} \alpha^{\mathrm{iC}}$, $\mathrm{H} \beta^{\mathrm{iC}}$, and $[\mathrm{OII}]^{\mathrm{iC}}$.

Finally, another way to estimate the dust attenuation is to compare the observed colors of the galaxies to a library of stellar-population synthesis models (hereafter SED-fitting). This work has been carried on with Bruzual \& Charlot (2003) models of SDSS galaxies by Kauffmann et al. (2003b), who provide an estimate of the continuum attenuation in the $z$-band $\left(A_{z}\right)$ with Charlot \& Fall (2000) attenuation curve. It is straightforward to convert the $A_{z}$ parameter to $\tau_{V}$ using the following formula:

$\tau_{V}^{\mathrm{SED}}=\frac{A_{z}}{1.086} \cdot\left(\frac{5500}{8800}\right)^{-0.7} \cdot \frac{1}{A_{\star} / A_{\mathrm{g}}}$.

The 1.086 factor is the conversion from magnitudes to opacities and $A_{\star} / A_{\mathrm{g}}$ is the mean ratio between the dust attenuation affecting stars (which dominate the colors of the galaxies) and the one affecting gas. The dust attenuation estimated from SED-fitting is characterized by a high uncertainty but might be used in a statistical way.

\subsection{Sample selection}

Thanks to the spectral classification provided by Kauffmann et al. (2003a), we selected only star-forming galaxies and removed all AGN from our sample. In the whole study, we also removed all galaxies for which $\mathrm{H} \alpha$ and $\mathrm{H} \beta$ emission lines (used for estimating the dust attenuation) are not measured with a signalto-noise ratio of at least 5 . We apply the same selection to the [OII] emission line in order to derive the calibrations in which this line is involved. The signal-to-noise ratios have been corrected according to the values provided in the SDSS/MPA website (based on the analysis of duplicated observations): they have been divided by 2.473 for $\mathrm{H} \alpha$ line, 1.882 for $\mathrm{H} \beta$ line and 2.199 for [OII] line. We also kept only galaxies with an available esti-

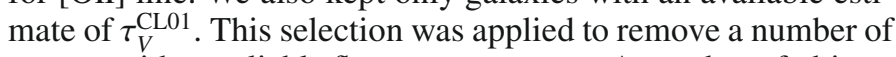
spectra with unreliable flux measurements. A number of objects may show a negative $\tau_{V}^{\text {Balmer }}$ estimate. This is due to statistical variations around the wrong assumption of a constant intrinsic Balmer ratio: these objects do not have to be removed.

Finally, some objects in the SDSS DR4 catalog were observed twice or more. We decided to keep only one observation of each duplicated galaxy by selecting the one with the best signal-to-noise ratio on the $\mathrm{H} \alpha$ emission line. We end up
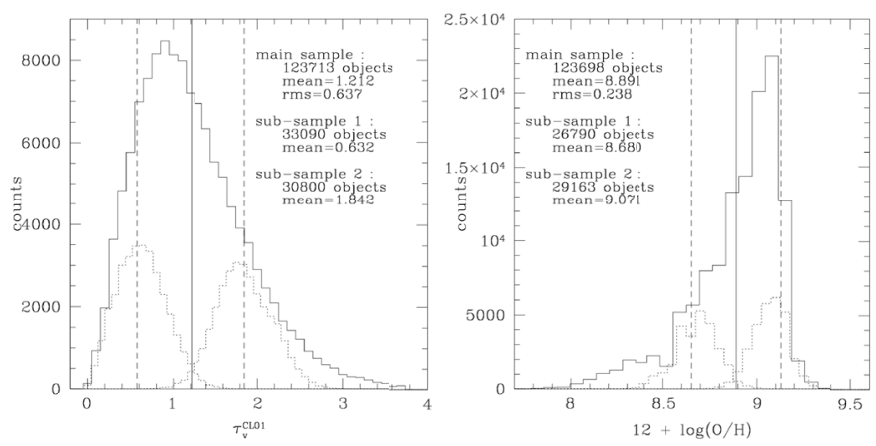

Fig. 1. This plot shows the histogram of the dust attenuation (left) or of the gas-phase oxygen abundance (right), both estimated with the CL01 method, for the starforming galaxies in the SDSS DR4 data. The solid vertical line shows the mean value of the distribution, and the dashed vertical lines the 1- $\sigma$ tails. The values are given in the plot. The two dotted histograms show the distribution of our two subsamples (see text for details).

with 123713 and 84733 starforming galaxies for $\mathrm{H} \alpha$-based and [OII]-based studies, respectively.

All the parameters used in this study (SFR, metallicity, dust attenuation) are the median estimates.

\subsection{Properties of the studied sample}

Figure 1 (left) shows the distribution of the effective dust attenuation in the $V$-band (estimated with the CL01 method) in the sample we have obtained. The mean value is 1.21 and the dispersion is 0.637 . We note that these values are only obtained with the selection of $\mathrm{H} \alpha$ and $\mathrm{H} \beta$ lines. The new mean and dispersion when we add the selection of the [OII] line are 1.10 and 0.547 , respectively. Figure 1 (right) shows the distribution of the gasphase oxygen abundance in the same sample (for objects with an available measurement of this parameter). The mean value is 8.89 with a dispersion of 0.24 . An 8.88 mean value with a dispersion of 0.21 if the selection on the [OII] line is applied.

Figure 1 also shows the distributions of four subsamples (two in each panel). These subsamples were randomly selected from the main sample, forcing their mean values to be closest as possible to the mean value of the main sample plus or minus its dispersion, and their dispersion to be half the dispersion of the main sample. We end up with four subsamples: low dust attenuation, high dust attenuation, low metallicity, and high metallicity. They will be used to test the calibrations on samples with different dust properties.

\section{SFR calibration with an available dust estimate}

We present in this section emission-line calibrations of the SFR based on data already corrected for dust attenuation. We first focus in Sect. 3.2 on data with dust attenuation estimated from the Balmer decrement, which is the common case in the literature. We then study in Sect. 3.3 the different calibrations we obtained when using the dust attenuation estimated from the CLO1 method or SED-fitting. 


\subsection{Base theory}

The common way to calibrate the SFR against one emission-line luminosity (e.g. $\mathrm{H} \alpha$ ) is a simple scaling relation of the following form:

$$
S F R=L(\mathrm{H} \alpha) / \eta_{\mathrm{H} \alpha} .
$$

This formula, where $\eta_{\mathrm{H} \alpha}$ is the efficiency factor, reproduces the simple relation between the amount of ionizing photons (the SFR) and the emission-line luminosity. However, it has already been shown that the $\mathrm{H} \alpha$ efficiency factor depends on other physical properties of the galaxies. Charlot \& Longhetti (2001) show that $\eta_{\mathrm{H} \alpha}$ depends on the metallicity, metal-rich stars being less luminous and thus producing less $\mathrm{H} \alpha$ photons in the interstellar medium for the same SFR. More recently, Brinchmann et al. (2004) show that $\eta_{\mathrm{H} \alpha}$ is also linked to the stellar mass of SDSS galaxies, massive galaxies producing less $\mathrm{H} \alpha$ photons than dwarfs for a given SFR. These two results are obviously linked by the mass-metallicity relation (Tremonti et al. 2004; Lamareille et al. 2008). Fortunately, Brinchmann et al. (2004) also show that this effect is canceled by the commonly used wrong assumption of a constant intrinsic $\mathrm{H} \alpha / \mathrm{H} \beta$ intrinsic Balmer ratio. In fact this ratio increases with metallicity, ending up with an overestimate of the dust attenuation for metal-rich galaxies. This effect compensates almost exactly for the decrease in $\eta_{\mathrm{H} \alpha}$ when metallicity increases.

Nevertheless, we decided to adopt a more general approach using a powerlaw as described by the following formula:

$$
\log (\mathrm{SFR})=\epsilon_{\mathrm{H} \alpha} \log L(\mathrm{H} \alpha)-\log \left(\eta_{\mathrm{H} \alpha}\right)
$$

The parameter $\epsilon_{\mathrm{H} \alpha}$ is the exponent of the power law. The simple calibration described by Eq. (5) corresponds to the $\epsilon_{\mathrm{H} \alpha}=1$ special case. We show in the following sections that this more general approach is not mandatory for the cases with a dust attenuation estimated from the Balmer decrement $\left(\epsilon_{\mathrm{H} \alpha}\right.$ is very close to 1 ), but that it is useful in other cases. We note that the correlation between dust attenuation and metallicity (Cortese et al. 2006; Mouhcine et al. 2005) also has an effect on the slope; however, the goal of our work is not to study in details such phenomenon.

In the following sections, the efficiency factors are expressed in $\operatorname{erg~s}^{-1}\left(M_{\odot} \mathrm{yr}^{-1}\right)^{-1}$ units and the exponents of the power laws are unitless. All fits are least square fits with errors in $x$ and $y$. The errors on the fitted parameters are given by the rms of 40 bootstrap estimates. The term "dispersion" relates to the rms of the residuals around the fitted calibrations, the term "shift" to the mean of these residuals. The given residual slopes are unitless.

\subsection{Dust estimated from the Balmer decrement}

We now present possible calibrations of the SFR based on data corrected with dust estimated from the Balmer decrement (see Eq. (3)). We remind the reader that the results presented in this subsection are only valid if we correct the observed flux for dust attenuation using Eqs. (2) and (3), i.e. making the wrong assumption that the intrinsic Balmer ratio is constant over the whole range of stellar masses.

\subsubsection{Improved standard calibrations}

Kennicutt (1998) has developed two calibrations of the SFR versus $\mathrm{H} \alpha^{\mathrm{i}}$ or the $[\mathrm{OII}]^{\mathrm{i}}$ emission lines, which are now widely used
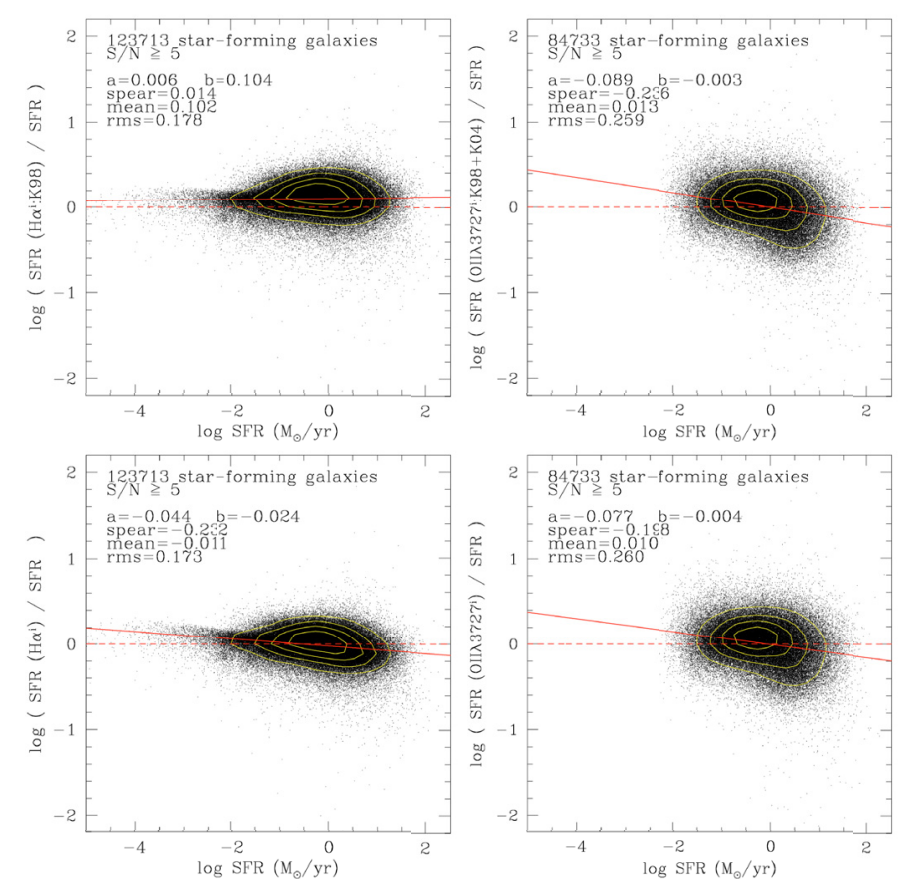

Fig. 2. These four plots show the residuals of the SFR recovered from an emission-line calibration as compared to the reference CL01 SFR, for starforming galaxies in the SDSS DR4 data. This comparison is shown as the logarithm of the ratio between the derived and the reference SFR, as a function of the logarithm of the reference SFR (in $M_{\odot} / \mathrm{yr}$ ). The number of objects used in the comparison, the shift, the dispersion, and the Spearman rank correlation coefficient are given in the plot. The dashed horizontal line is the $y=0$ curve, the solid line is the $y=a \cdot x+b$ curve where $a$ and $b$ are the parameters of the linear regression given in the plot. Isodensity contours are overplotted in white. The studied calibrations are: top-left: Kennicutt (1998) H $\alpha^{\mathrm{i}}$; top-right: Kennicutt (1998) (corrected by Kewley et al. 2004) [OII] $]^{\mathrm{i}}$; bottom-left: our improved $\mathrm{H} \alpha^{\mathrm{i}}$ calibration; bottom-right: our improved [OII] ${ }^{\mathrm{i}}$ calibration.

in the literature. We take advantage of the SDSS DR4 data to test these two calibrations as compared to the high-quality CL01 estimate of the SFR. We also derive two new improved calibrations based on Eq. (6) rather than Eq. (5).

Figure 2 (top-left) shows the comparison between the CL01 SFR and the one recovered with the standard Kennicutt (1998) $\mathrm{H} \alpha^{\mathrm{i}}$ calibration, which uses $\log \left(\eta_{\mathrm{H} \alpha}^{\mathrm{i}}\right)=41.28$ if we scale their results to our adopted IMF. We find good agreement: the dispersion is $0.17 \mathrm{dex}$, which is very similar to the minimum uncertainty associated to the CL01 SFR estimates (i.e. 0.16 dex). Thus, we confirm that the Ho emission line luminosity alone is sufficient to recover the SFR almost perfectly.

However, we see that the reduced efficiency factor and the dust attenuation overestimate do not exactly cancel each other on the massive end of the sample, which induces a mean of the residuals of +0.1 dex. One might decide to subtract 0.1 dex to the Kennicutt (1998) calibration in order to find a null mean of the residuals, or we can try to derive an improved calibration.

Figure 3 (left) shows our improved calibration for the correlation between the $\mathrm{H} \alpha^{\mathrm{i}}$ emission-line luminosity and the SFR in SDSS DR4 data. We find the following best fit values:

$\left\{\begin{aligned} \log \eta_{\mathrm{H} \alpha}^{\mathrm{i}} & =39.38 \pm 0.03 \\ \epsilon_{\mathrm{H} \alpha}^{\mathrm{i}} & =0.951 \pm 0.001\end{aligned}\right.$

One can notice that the formal errors of these two parameters are very low thanks to the large number of objects, which makes this 

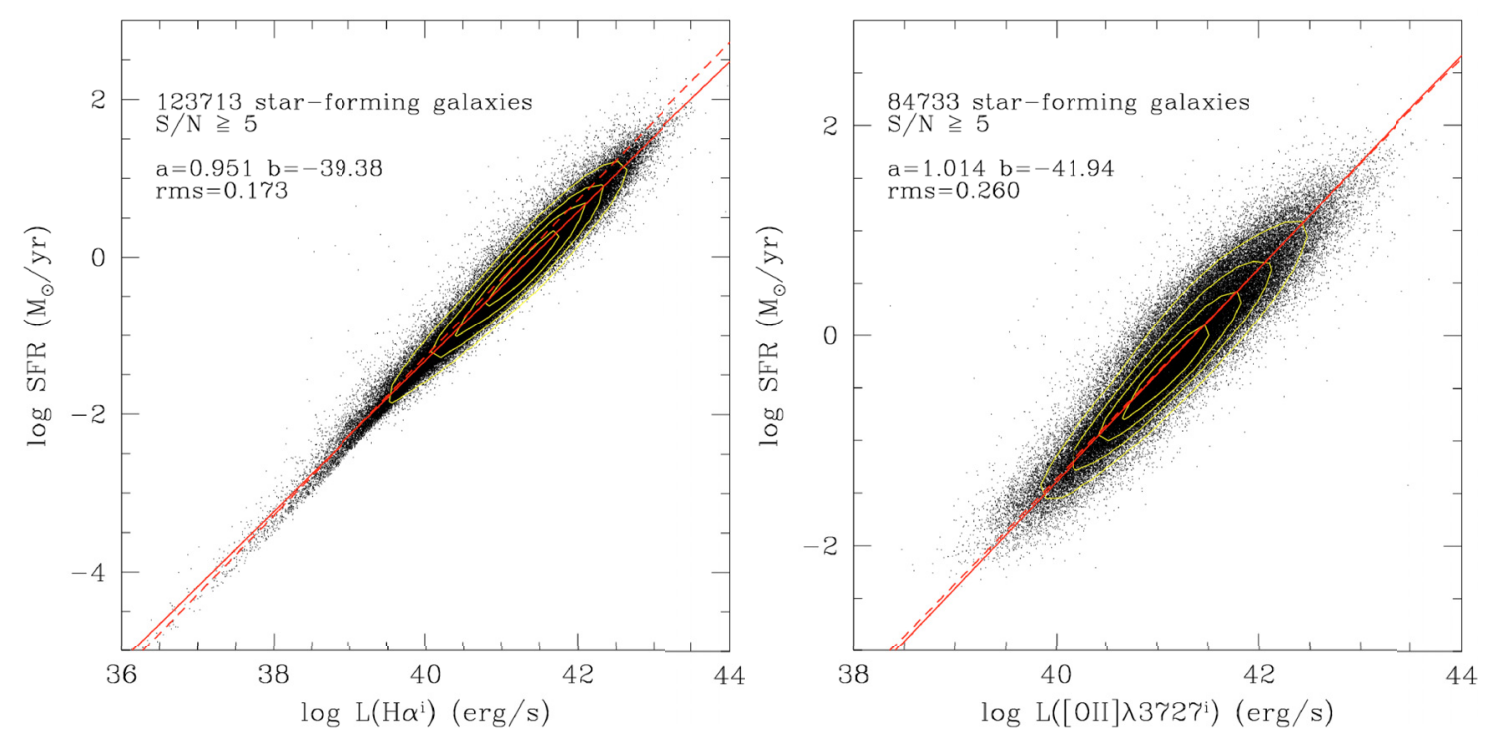

Fig. 3. Relation between the SFR (logarithm of $M_{\odot} / \mathrm{yr}$ ) and the $\mathrm{H} \alpha^{\mathrm{i}}($ left $)$ or [OII] $]^{\mathrm{i}}$ (right) emission lines luminosities (logarithm of erg/s) for starforming galaxies in the SDSS DR4 data. The solid line is a least-square fit to the data (errors on $x$ and $y$ ). The dashed line is the Kennicutt (1998) calibration corrected to our assumed IMF, and corrected for dust by Kewley et al. (2004) in the right panel. Isodensity contours are overplotted in white.

calibration quite reliable. The uncertainty on the exponent of the power law especially tells us that this parameter is very well constrained, while not equal to 1 as compared to all previous studies. As shown in Fig. 2 (bottom-left), the mean on the residuals is now almost null (0.01 dex) with the improved calibration. A small residual slope $(-0.04)$ is present as the improved calibration is now less accurate for very low SFR values. This residual slope is not significant given the low Spearman rank correlation coefficient: -0.23 .

We now consider the case where the $\mathrm{H} \alpha$ line is not observed, or not measurable, and that we decide to use the [OII] $\lambda 3727$ emission line instead. In such case, the dust attenuation might be derived from another Balmer ratio not involving the $\mathrm{H} \alpha$ line (e.g. $\mathrm{H} \gamma / \mathrm{H} \beta, \mathrm{H} \delta / \mathrm{H} \gamma, \ldots$ )

As already stated in the literature, the relation between the SFR and the [OII] line has to be considered with caution because of the stronger dependence of this line on metallicity (compared to $\mathrm{H} \alpha$ ), as well as a stronger sensibility to dust attenuation. However, as shown in Fig. 2 (top-right), we find a good agreement with the standard Kennicutt (1998) [OII] ${ }^{\mathrm{i}}$ calibration, which uses $\log \left(\eta_{[\mathrm{OII}]}^{\mathrm{i}}\right)=41.36$ if we correct their results for dust attenuation (Kewley et al. 2004) and scale them to our adopted IMF.

It seems that the effect of metallicity on the [OII] line does not produce any systematic shift and only leads to a higher dispersion: 0.25 dex. To be more precise, the three effects of the metallicity on the relative strength of the [OII] line, on the efficiency factor, and on the estimate of the dust attenuation exactly compensate in order to produce a exponent of the power law very close to unity.

Figure 3 (right) shows our improved calibration in the correlation between the $[\mathrm{OII}]^{\mathrm{i}}$ emission-line luminosity and the SFR in SDSS DR4 data. We find the following best-fit values:

$\left\{\begin{array}{cl}\log \eta_{\text {[OII] }}^{\mathrm{i}} & =41.94 \pm 0.7 \\ \epsilon_{\text {[OII] }}^{\mathrm{i}} & =1.014 \pm 0.02\end{array}\right.$

As expected, and as shown in Fig. 2 (bottom-right), this calibration does not produce any significant improvement compared to the Kennicutt (1998) (corrected by Kewley et al. 2004) calibration. In both cases, the observed residual slope of -0.09 is not significant (Spearman rank correlation coefficient of -0.24).

\subsubsection{New calibrations}

As already shown in previous works (Kewley et al. 2004; Moustakas et al. 2006; Weiner et al. 2007), the best way to provide a reliable SFR vs. [OII] calibration is to actually use the $\mathrm{SFR}$ vs. $\mathrm{H} \alpha^{\mathrm{i}}$ calibration, and to calibrate either the $[\mathrm{OII}] / \mathrm{H} \alpha^{\mathrm{i}}$ (observed-to-intrinsic) or the $[\mathrm{OII}]^{\mathrm{i}} / \mathrm{H} \alpha^{\mathrm{i}}$ (intrinsic-to-intrinsic) line ratio against a well chosen parameter. Then the star formation rate is given by the following formula:

$\log \mathrm{SFR}=\epsilon_{\mathrm{H} \alpha}^{\mathrm{i}}\left(\log L\left([\mathrm{OII}]^{\mathrm{i}}\right)-\log \left([\mathrm{OII}]^{\mathrm{i} ?} / \mathrm{H} \alpha^{\mathrm{i}}\right)\right)-\log \eta_{\mathrm{H} \alpha}^{\mathrm{i}}$

Kewley et al. (2004) has calibrated the $[\mathrm{OII}]^{\mathrm{i}} / \mathrm{H} \alpha^{\mathrm{i}}$ line ratio against the gas-phase oxygen abundance $(12+\log (\mathrm{O} / \mathrm{H}))$, ending up in an appreciable improvement of the calibration. As also shown by Mouhcine et al. (2005) with 2dFGRS data and in Fig. 4, there is indeed a robust correlation between these two parameters. The dashed line in Fig. 4 shows the relation derived semi-empirically by Kewley et al. (2004). The residuals around this relation are characterized by a mean of -0.04 dex and a rms of 0.1 dex in SDSS DR4 data.

However, we see three drawbacks against the use of this relation:

- first the gas-phase oxygen abundance is not an easy parameter to derive. There are many different calibrations which can be different from each other by up to 0.5 dex (see Kewley \& Ellison 2008, for a detailed discussion). Moreover, most of these calibrations need many emission lines to be measured and we emphasize that, in such cases, it might be easier to use directly the SFR estimated from multiple-lines fitting methods like CLO1;

- second, we already stated before that correcting the [OII] emission line for dust attenuation requires in most common cases that the $\mathrm{H} \alpha$ emission line is being observed: in this case one would estimate the SFR directly from the Ho line, rather 


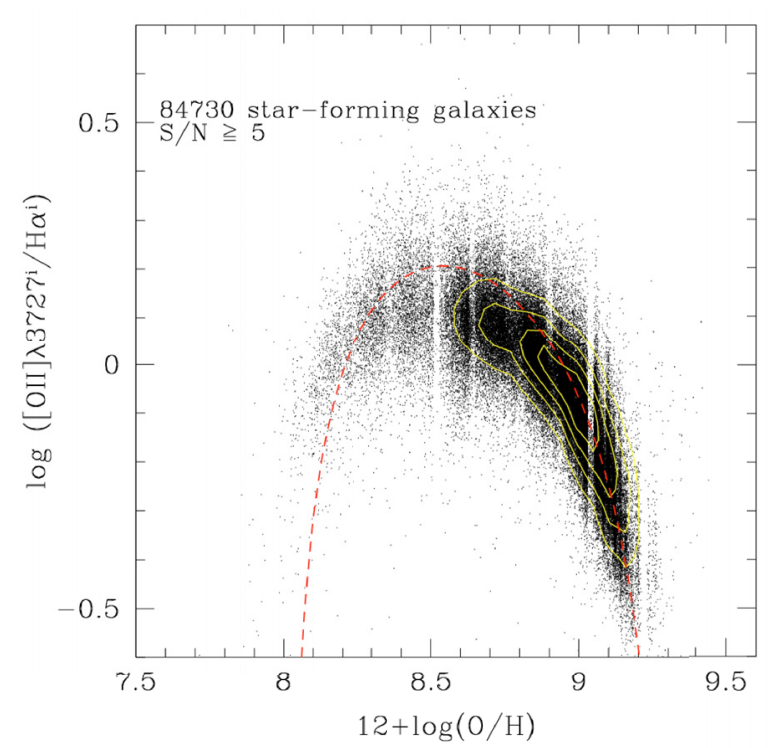

Fig. 4. Relation between the $[\mathrm{OII}] \lambda 3727^{\mathrm{i}} / \mathrm{H} \alpha^{\mathrm{i}}$ emission line ratio and gas-phase oxygen abundance (estimated with the CL01 method) for the star-forming galaxies in the SDSS DR4 catalog. The dashed curve is a semi-empirical estimation of this relation (Kewley et al. 2004).
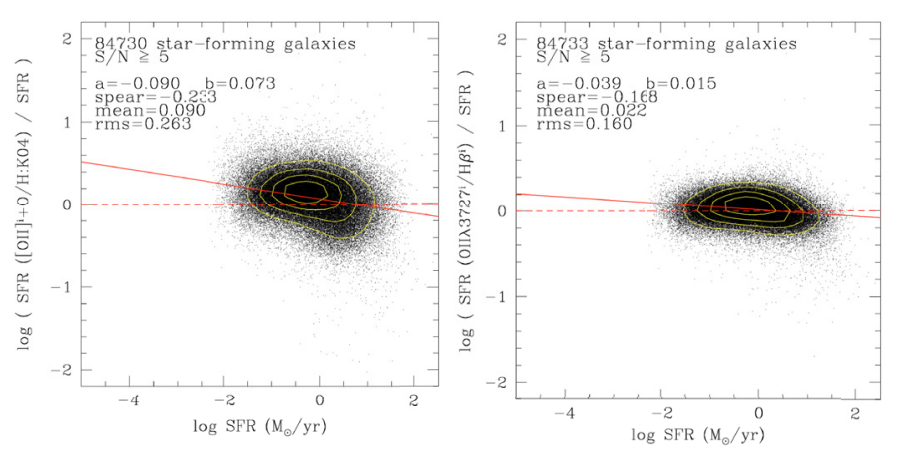

Fig. 5. Same legend as in Fig. 2. The studied calibrations are: left: Kewley et al. (2004) [OII] ${ }^{\mathrm{i}}$ with a correction for metallicity; right: our new $[\mathrm{OII}]^{\mathrm{i}}+\mathrm{H} \beta^{\mathrm{i}}$ calibration.

from the [OII] line. Thus, trying to improve the [OII] calibration with data corrected for dust attenuation is actually not applicable in most common cases;

- for the other cases (e.g. dust attenuation estimated from another Balmer ratio), we also note that using the metallicity estimate (or any other derived parameter) to correct the calibration is not necessary. Indeed a very simple relation, which do not need a metallicity estimate, already exists between [OII $]^{\mathrm{i}}, \mathrm{H} \alpha^{\mathrm{i}}$ and $\mathrm{H} \beta^{\mathrm{i}}$ emission lines. This simple relation is expressed by the following equation assuming the standard intrinsic Balmer ratio:

$$
\frac{[\mathrm{OII}]^{\mathrm{i}}}{\mathrm{H} \alpha^{\mathrm{i}}}=\frac{[\mathrm{OII}]^{\mathrm{i}}}{\mathrm{H} \beta^{\mathrm{i}}} / \frac{\mathrm{H} \alpha^{\mathrm{i}}}{\mathrm{H} \beta^{\mathrm{i}}}=\frac{[\mathrm{OII}]^{\mathrm{i}}}{\mathrm{H} \beta^{\mathrm{i}}} / 2.85
$$

We applied Eq. (15) of Kewley et al. (2004) in order to compare their metallicity- and dust-corrected calibration to the reference CL01 SFR. We have done that only for the 84730 galaxies for which an estimate of the gas-phase oxygen abundance was available. Before doing that, we have corrected our available CL01 metallicities to Kewley \& Dopita (2002) metallicities, using the formula in Table B3 of Kewley \& Ellison (2008).

As shown in Fig. 5 (left), the Kewley et al. (2004) calibration shows a non-negligible 0.1 dex shift. Moreover, the dispersion of the relation is 0.26 dex, which is not better than the standard $[\mathrm{OII}]^{\mathrm{i}}$ calibration without a correction for metallicity. Their is also a small, not significant, residual slope of -0.09 (Spearman rank correlation coefficient of -0.23$)$.

Equations (9) and (10) are now combined to provide an estimate of the SFR, which is compared to the CL01 SFR in Fig. 5 (right). The relation is, as expected, very good as no dispersion is added by Eq. (10). The small dispersion of the relation ( 0.16 dex) only comes from the uncertainty of the $\mathrm{H} \alpha^{\mathrm{i}}$ calibration that we derived previously. This calibration does not show any significant residual slope (Spearman rank correlation coefficient: -0.17$)$. In fact, this new [OII $]^{\mathrm{i}}+\mathrm{H} \beta^{\mathrm{i}}$ calibration is expected to give exactly the same results as the underlying $\mathrm{H} \alpha^{\mathrm{i}}$ calibration since the relation between them is the constant 2.85 factor. Figure 5 (right) is nothing else than Fig. 2 (bottom-left) without some missing points because of the additional selection of the [OII] line. These missing points explain the smaller scatter.

Following this conclusion, we emphasize that a simple $\mathrm{H} \beta^{\mathrm{i}}$ calibration is even easier to derive and to apply to the observations using this relation:

$\mathrm{H} \alpha^{\mathrm{i}}=\mathrm{H} \beta^{\mathrm{i}} \times 2.85$

The $\mathrm{H} \beta^{\mathrm{i}}$ is expected to give exactly the same results as the $\mathrm{H} \alpha^{\mathrm{i}}$ calibration, provided that a correction for dust attenuation has been estimated from the assumption of a constant intrinsic Balmer ratio.

\subsection{Dust estimated from another method}

The dust attenuation might not be estimated with the assumption of a constant intrinsic Balmer decrement. Other methods such as CL01 can provide a better estimate of the dust attenuation, not biased towards the metallicity dependence of the intrinsic Balmer ratio (thanks to the use of all available emission-line measurements). The dust attenuation estimated from SED fitting methods does not either make the assumption of a constant intrinsic Balmer ratio.

As stated before, the calibrations derived in previous subsection should show an exponent of the power law greater than 1.0, because of the metallicity dependence of the relation between the SFR and the emission-line luminosities (assuming that the higher is the SFR the higher are the stellar mass and the metallicity). However, this effect is not seen since it is almost exactly compensated by the overestimate of the dust attenuation at high metallicities.

We thus study in this subsection the results obtained with dust estimated from other methods.

\subsubsection{Metallicity-unbiased calibrations}

We provide new calibrations to be used on data corrected with a good estimate of the dust attenuation, i.e. not biased towards metallicity. To this end, we corrected the emission-line luminosities using the dust attenuation estimated with the CL01 method, instead of the one estimated from the Balmer decrement. We emphasize that this work has mainly been done for comparison purposes: using the CL01 method to estimate the dust attenuation makes our SFR calibration useless since the CL01 SFR is already better constrained.

Figure 6 shows the relations between the SFR and $\mathrm{H} \alpha^{\mathrm{iC}}$, [OII $]^{\mathrm{iC}}$, or $\mathrm{H} \beta^{\mathrm{iC}}$ emission lines. In the three cases, we find as expected an exponent of the power law greater than 1 . However, we see that the metallicity dependence of the efficiency factor 

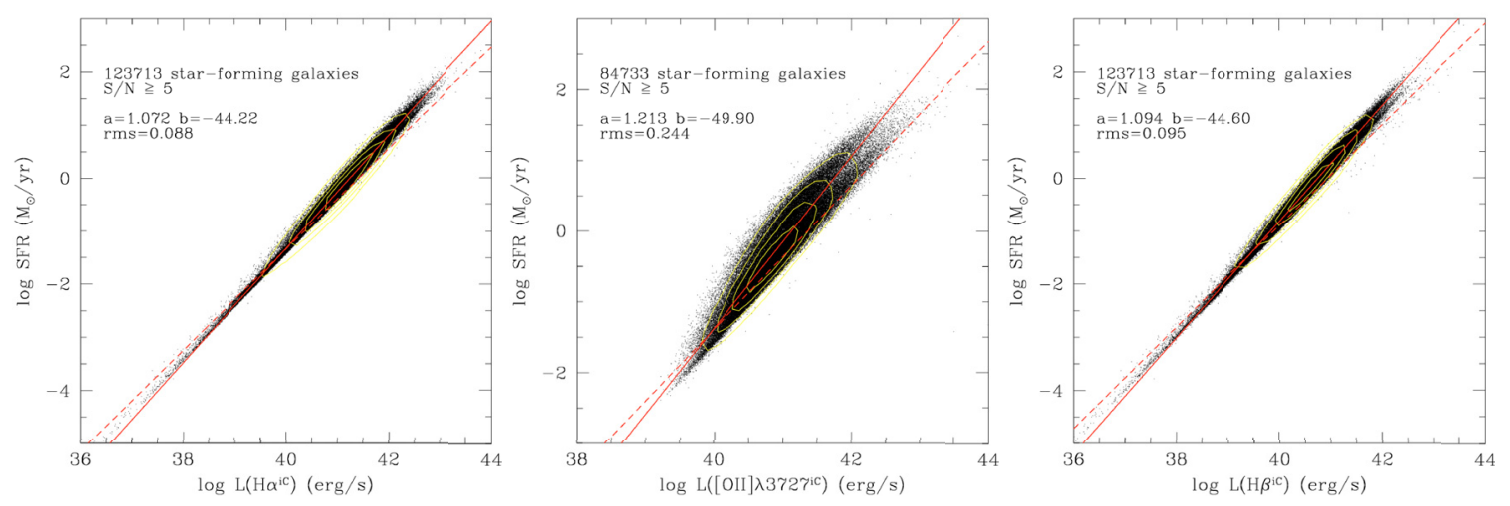

Fig. 6. Relation between the SFR (logarithm of $M_{\odot} / \mathrm{yr}$ ) and the $\mathrm{H} \alpha^{\mathrm{iC}}(\text { left), [OII }]^{\mathrm{iC}}$ (center), or $\mathrm{H} \beta^{\mathrm{iC}}$ emission-line luminosities (logarithm of erg/s) for starforming galaxies in the SDSS DR4 data. The solid line is a least-square fit to the data (errors on $x$ and $y$ ). These data have been corrected using the unbiased CL01 estimate of dust attenuation. The dashed lines show the previous calibrations obtained with dust estimated from the assumption of a constant intrinsic Balmer ratio (see Fig. 3). Isodensity contours are overplotted in white.

does not have a strong effect on the slope for the $\mathrm{H} \alpha^{\mathrm{iC}}$ and $\mathrm{H} \beta^{\mathrm{iC}}$ calibrations. We remind the reader that, in contrast to what we said in Sect. 3.2, the calibrations based on $\mathrm{H} \alpha^{\mathrm{iC}}$ or $\mathrm{H} \beta^{\mathrm{iC}}$ lines are now slightly different since the ratio between these two lines is no longer an assumed constant. We obtain the following results:

$$
\begin{aligned}
& \left\{\begin{array}{cl}
\log \eta_{\mathrm{H} \alpha}^{\mathrm{iC}} & =44.22 \pm 0.03 \\
\epsilon_{\mathrm{H} \alpha}^{\mathrm{iC}} & =1.072 \pm 0.001
\end{array}\right. \\
& \left\{\begin{array}{cl}
\log \eta_{[\mathrm{OII}]}^{\mathrm{iC}} & =49.90 \pm 0.9 \\
\epsilon_{[\mathrm{OII}]}^{\mathrm{iC}} & =1.213 \pm 0.03
\end{array}\right. \\
& \left\{\begin{array}{cc}
\log \eta_{\mathrm{H} \beta}^{\mathrm{iC}} & =44.60 \pm 0.03 \\
\epsilon_{\mathrm{H} \beta}^{\mathrm{iC}} & =1.094 \pm 0.001 .
\end{array}\right.
\end{aligned}
$$

In the three cases, the dispersion is reduced thanks to the better estimate of the dust attenuation provided by the CL01 method. It is now $<0.1$ dex in the $\mathrm{H} \alpha^{\mathrm{iC}}$ and $\mathrm{H} \beta^{\mathrm{iC}}$ calibrations and 0.24 dex in the $[\mathrm{OII}]^{\mathrm{iC}}$ calibration.

\subsubsection{Dust estimated from SED fitting}

One might ask if emission lines can also be used to derived SFR with an estimation of dust attenuation coming from SED fitting. Figure 7 (left) shows the correlation between the intrinsic $\mathrm{H} \alpha$ emission-line luminosity and the SFR in SDSS DR4 data, if we correct $\mathrm{H} \alpha$ luminosities with the dust attenuation computed from SED-fitting by Kauffmann et al. (2003b) (see Eq. (4)). Only the 87213 galaxies with an available estimate of the dust attenuation are plotted.

We used a stellar-to-gas attenuation ratio of 0.3 that corresponds to the mean value observed in the SDSS data (Brinchmann, private communication). We see that the exponent of the power law is much smaller $(0.710 \pm 0.001)$, telling us that the SED-fitting dust attenuation is even more overestimated at higher masses than with the Balmer decrement method.

But no strong conclusion can be drawn: the dispersion that we find $(0.40 \mathrm{dex})$ is indeed quite high; moreover, it does not take the likely variations in the stellar-to-gas attenuation ratio into account between the SDSS data and any other sample. Using instead a stellar-to-gas attenuation ratio of 0.44 (Calzetti 2001), we found in Fig. 7 (right) an exponent of the power law of $0.864 \pm 0.001$. The derived efficiency factors with a mean stellar-to-gas attenuation ratio of 0.3 and 0.44 are respectively $29.67 \pm 0.02$ and $35.79 \pm 0.03$. We emphasize that a stellar-to-gas
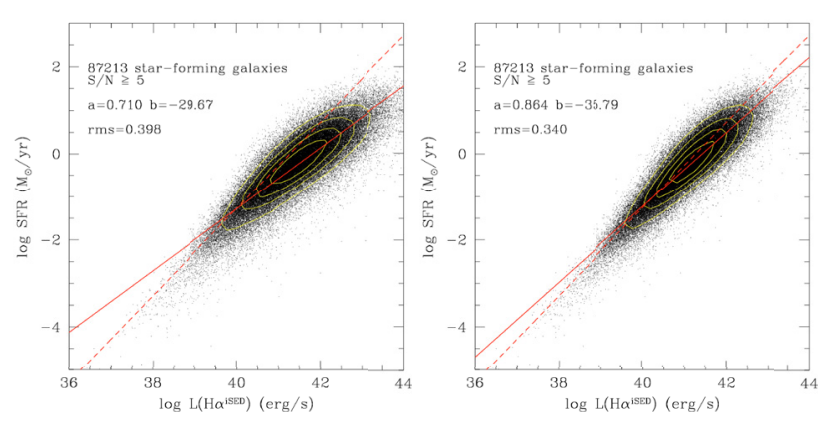

Fig. 7. Relation between the SFR (logarithm of $M_{\odot} / \mathrm{yr}$ ) and the $\mathrm{H} \alpha^{\mathrm{iSED}}$ emission lines luminosity (logarithm of erg/s) for star-forming galaxies in the SDSS DR4 data. The solid line is a least-square fit to the data (errors on $x$ and $y$ ). The data has been corrected for dust attenuation estimated with the SED fitting method (see Eq. (4)). The stellar-to-gas attenuation ratio has been assumed to 0.3 (left: SDSS mean value), or 0.44 (right: Calzetti 2001). The dashed line is the Kennicutt (1998) calibration corrected to our assumed IMF. Isodensity contours are overplotted in white.

attenuation ratio of 0.44 is not the right value for representing these data, even if it by chance gives a less dispersed calibration $(0.34$ dex $)$.

We conclude that the most critical issue in using dust estimated from SED-fitting, in order to correct emission lines, comes from the uncertainty in the stellar-to-gas attenuation ratio. This problem makes SFR derived using this method almost completely random on a galaxy per galaxy basis. This method might be used in a statistical way, but with great uncertainties coming both from the dispersion observed in Fig. 7, and from the fact that one has to know the right stellar-to-gas attenuation ratio to apply to the data.

\section{SFR calibration without a dust estimate}

We present in this section emission-line calibrations of the SFR based on data not corrected for dust attenuation. Because of the small wavelength coverage of many spectroscopic surveys, it is common that not all emission lines needed to derive a correction for dust attenuation and to compute an SFR are present in a given spectrum.

There are two ways to handle such data: (i) use an assumed mean extinction and apply a statistical correction for dust attenuation (see Sect. 4.1); or (ii) use an SFR calibration which 

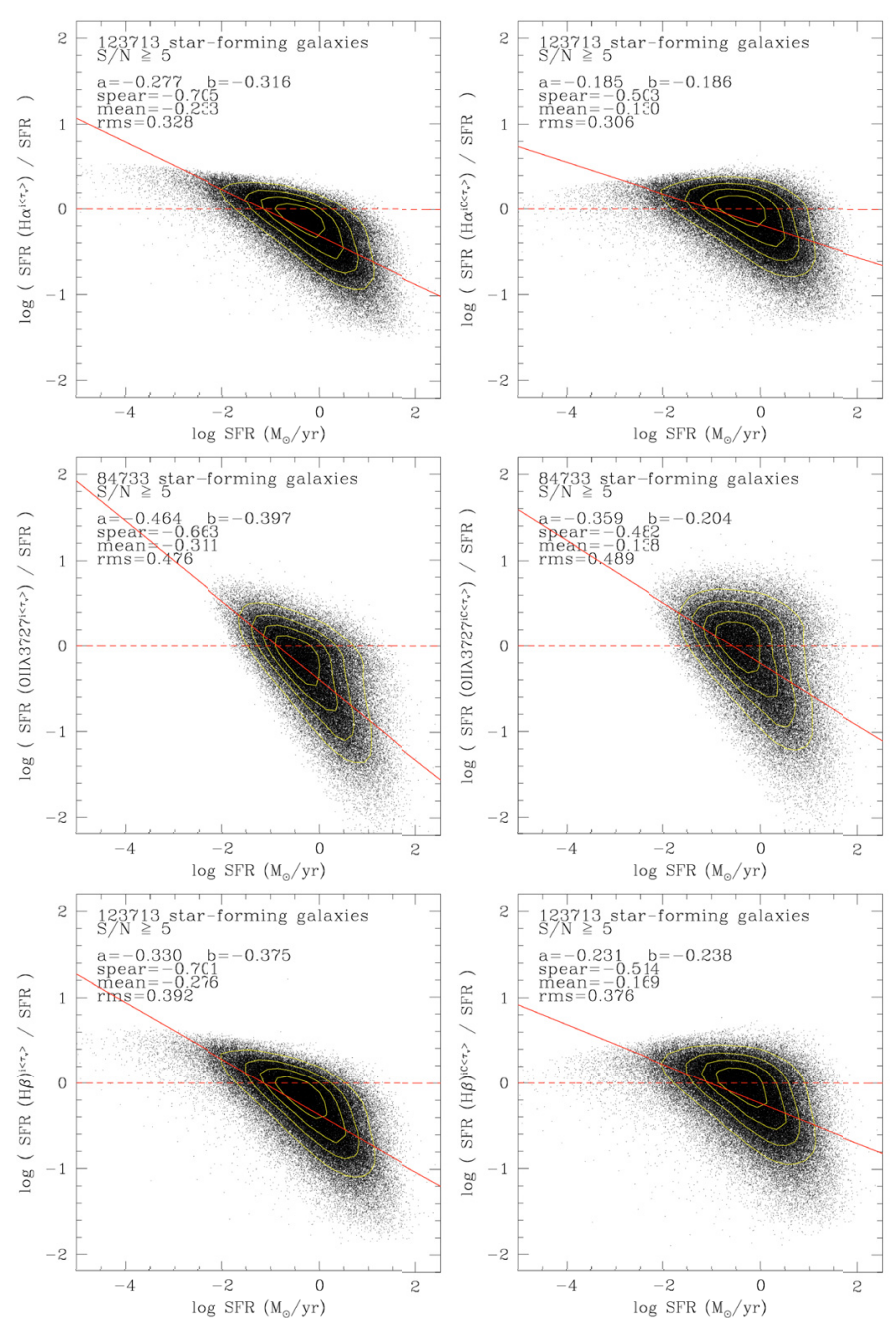

Fig. 8. Same legend as in Fig. 2. The calibrations are the standard $\mathrm{H} \alpha^{\mathrm{i}}$ (top-left), [OII $]^{\mathrm{i}}$ (middle-left), and $\mathrm{H} \beta^{\mathrm{i}}$ (bottom-left) calibrations, or the metallicity-unbiased $\mathrm{H} \alpha^{\mathrm{iC}}$ (top-right), [OII ${ }^{\mathrm{iC}}$ (middle-right), and $\mathrm{H} \beta^{\mathrm{iC}}$ (bottom-right) calibrations, all applied to data corrected using an assumed mean dust attenuation $A_{V}=1$.

provides, still in a statistical approach, a self-consistent correction for dust-attenuation, i.e. with dust properties recovered from another parameter (see Sect. 4.2). Such self-consistent correction depends on the properties of the sample used to do the calibration. Thus, we present a way to correct for this bias in Sect. 4.3.

\subsection{Use of an assumed mean correction}

When dust attenuation cannot be reliably derived from the data, it is common in the literature to use an assumed mean correction (frequently $A_{V}=1$ ).

\subsubsection{Standard calibrations with $A_{V}=1$}

In this subsection, we discuss the quality of the standard calibrations used on SDSS DR4 with an assumed mean correction for dust of $A_{V}=1$.

One important question we need to answer is what kind of calibration we should use on data corrected with a mean dust attenuation. Should we use the standard calibrations derived with the wrong assumption of a constant intrinsic Balmer ratio (Sect. 3.2) or our new calibrations derived with the better

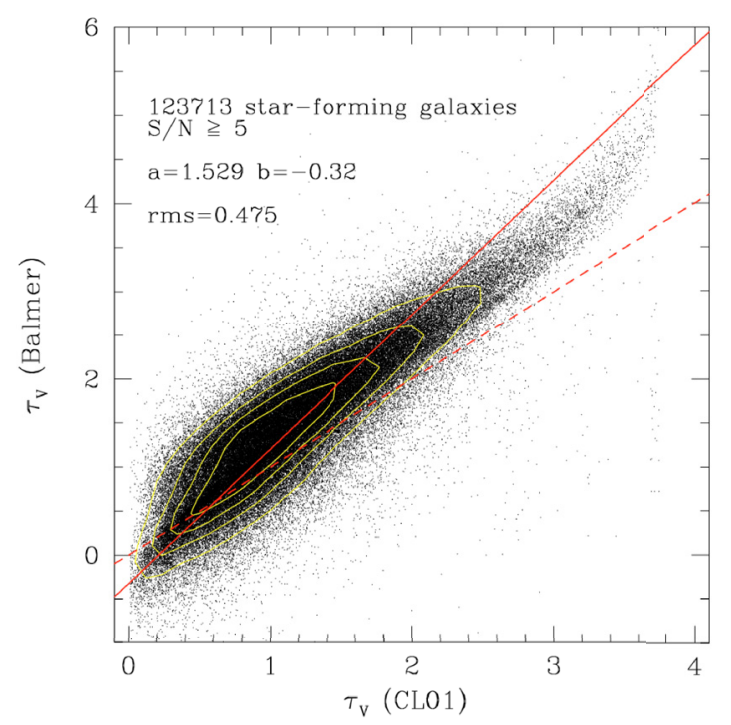

Fig. 9. Relation between the dust attenuation estimated with the CL01 method or with the Balmer decrement method, for starforming galaxies in the SDSS DR4 data. The dashed line is the $y=x$ curve. The solid line is the $y=a \cdot x+b$ curve where $a$ and $b$ are the parameters of the least-square fit (errors in $x$ and $y$ ), as given in the plot. Isodensity contours are overplotted in white.

CL01 estimates of the dust (Sect. 3.3)? The answer basically depends on the way the mean dust attenuation has been estimated (is it biased towards the metallicity dependence of the intrinsic Balmer ratio or not?).

We thus plot the comparison of both calibrations in Fig. 8. The main conclusion we draw from this figure is that the residuals are not only highly dispersed (rms in the range 0.3-0.5 dex depending on the calibration) but also show a clear residual slope in all cases $(-0.2$ to -0.5 depending on the calibration). This residual slope is the signature of the correlation between the dust attenuation and the SFR in galaxies. This correlation is obviously not taken into account by the assumption of a constant dust attenuation for the whole sample.

The residual slopes are significant as shown by the Spearman-rank correlation coefficients $(-0.5$ to -0.7$)$. It is also clear that the residual slopes obtained with the $\mathrm{H} \alpha^{\mathrm{iC}}$, [OII ${ }^{\mathrm{iC}}$, and $\mathrm{H} \beta^{\mathrm{iC}}$ calibrations, based on the CL01 estimates of the dust, are not only smaller but also less significant (smaller Spearman rank correlation coefficient).

The method of the assumed mean correction thus leads to poor results, either from galaxy-per-galaxy studies (high dispersion) or from statistical studies (non-negligible residual slope). This method applied with the [OII] line is particularly inadvisable (worst dispersion and steeper residual slope).

\subsubsection{Additional sources of biases}

We now discuss the additional effect of using of a wrong mean correction. Following Eqs. (2) and (6), the bias introduced into the SFR can be computed as

$\log \left(\frac{\mathrm{SFR}^{\mathrm{w}}}{\mathrm{SFR}}\right)=\epsilon \times \frac{\tau_{V}^{\mathrm{w}}-\tau_{V}}{\ln (10)}$,

where $\tau_{V}^{\mathrm{w}}$ is the wrong assumed dust attenuation, and SFR ${ }^{\mathrm{w}}$ is the wrong derived SFR. Below, we investigate three possible sources of bias. 
The first bias comes from the assumption of $A_{V}=1$. The sample used in this study actually shows a mean dust attenuation $\left\langle\tau_{V}^{\mathrm{CL} 01}\right\rangle=1.21$ (see Sect. 2 and Fig. 1). Converting from opacities to magnitudes, we obtain $\left\langle A_{V}^{\mathrm{CL} 01}\right\rangle=\left\langle\tau_{V}^{\mathrm{CL} 01}\right\rangle \times 1.086=1.31$. The difference between the assumed $A_{V}=1$ and the actual value in the sample is partly responsible for the non-null means of the residuals observed in Fig. 8. When applying Eq. (15), we find a theoretical bias on the order of $-0.15 \mathrm{dex}$, which is similar to the observed values for the CL01 calibrations (Fig. 8 right).

The second bias comes from the dispersion in the distribution of dust attenuation. In the SDSS DR4 data, the dispersion on the derived dust attenuation is 0.64 , which means that the mean dust attenuation may not be assumed with a better precision than \pm 0.64 . Thus it implies a possible systematic shift of about \pm 0.30 dex. As stated in Sect. 2, the mean dust attenuation is $\left\langle\tau_{V}^{\mathrm{CL} 01}\right\rangle=1.10$ instead of 1.21 when we add the selection on the [OII] line, which implies a bias of about \pm 0.05 dex.

Finally, we remind the reader to think carefully about the origin of the assumed mean correction before applying it; i.e. he or she has to check whether this assumption comes from a dust attenuation derived with a constant Balmer decrement or from an unbiased estimation. Having checked that, the right calibrations could be applied. Figure 9 gives for convenience the relation between $\tau_{V}^{\text {Balmer }}$ and the unbiased $\tau_{V}^{\mathrm{CL} 01}$ in the SDSS DR4 data. We fit an empirical relation that follows this formula:

$\tau_{V}^{\mathrm{Balmer}}=1.53 \times \tau_{V}^{\mathrm{CL} 01}-0.3$.

This implies a theoretical bias on the order of -0.25 dex, which is again similar to the observed values for the standard calibrations (Fig. 8 left). Hence we conclude that the use of an assumed mean correction has to be handled with great care.

\subsection{Use of a self-consistent correction}

As stated in the previous section, using of an assumed mean correction has one main drawback: it does not take into account the variation in the dust attenuation as a function of SFR. We thus discuss in this section three possible ways to reduce this bias. The idea common to these three possibilities is that the relation between the dust attenuation and the SFR might itself be recovered from the data, i.e. self-consistently. We explore the self-consistent corrections obtained either from the observed line luminosities, another parameter like the magnitude, or a combination of two emission lines.

\subsubsection{Single-line calibrations}

The simplest way to apply a self-consistent correction for dust is to relate the dust attenuation to the line luminosity itself. Hence, it consists on calibrating the SFR directly with the observed, not intrinsic, line luminosity with a two-parameter law as described in Eq. (6).

Figure 10 (top-left) shows the results obtained when using the standard Kennicutt (1992) $\mathrm{H} \alpha^{\mathrm{i}}$ calibration on data uncorrected for dust attenuation. We clearly see that the dust attenuation causes an underestimate of the SFR (the mean of the residuals is $-0.48 \mathrm{dex}$ ) and that this effect increases with SFR. Moreover the dispersion is $0.32 \mathrm{dex}$, and there is a significant residual slope of -0.24 (Spearman rank correlation coefficient of -0.63). Taking this into account, a two parameter law is necessary, differently from what we observed with dust-corrected data in Sect. 3.2.

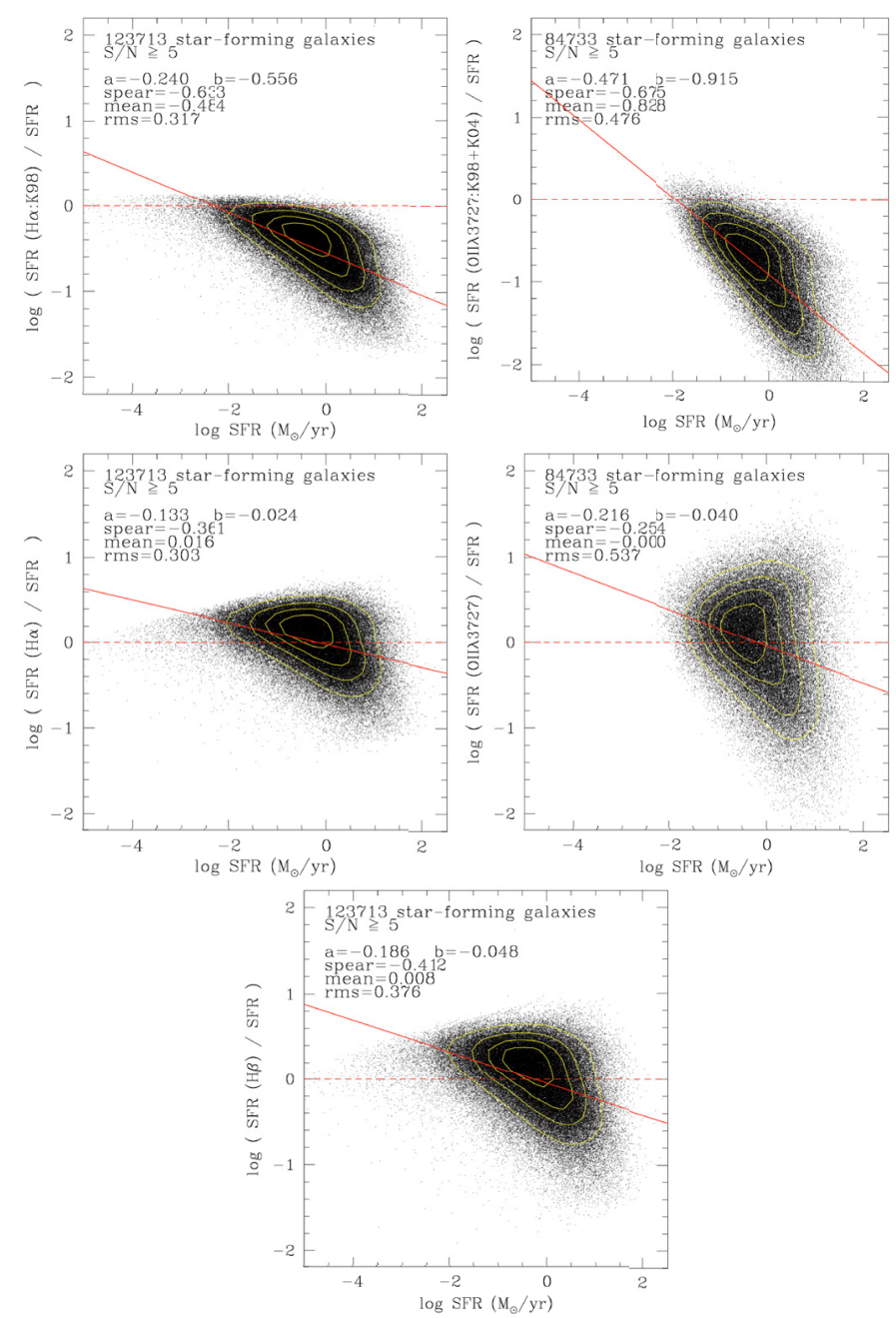

Fig. 10. Same legend as in Fig. 2. The calibrations are: top-left: Kennicutt (1998) H $\alpha^{\mathrm{i}}$ applied to uncorrected data; top-right: Kennicutt (1998) (corrected by Kewley et al. 2004) [OII] ${ }^{i}$ to uncorrected data; middle-left: our new $\mathrm{H} \alpha$ calibration; middle-right: our new [OII] calibration; bottom: our new $\mathrm{H} \beta$ calibration.

Figure 11 (left) shows the correlation between the observed $\mathrm{H} \alpha$ emission-line luminosity and the SFR in SDSS DR4 data. We obtain the following new calibration:

$\left\{\begin{array}{cl}\log \eta_{\mathrm{H} \alpha} & =46.49 \pm 0.04 \\ \epsilon_{\mathrm{H} \alpha} & =1.141 \pm 0.001 .\end{array}\right.$

As shown in Fig. 10 (middle-left), the dispersion of this new calibration is 0.30 dex, which tells us that the SFR recovered without dust attenuation correction are almost twice more dispersed than when an estimate of dust attenuation is available. The mean of the residuals is now almost zero (0.02 dex). This calibration is better, in terms of the dispersion, than the results obtained with an assumed mean correction (see Sect. 4.1, dispersion of $0.33 \mathrm{dex}$ ), but is still quite poor.

Unfortunately the use of the two-parameters law, which allows in principle taking the dependence into account between the dust attenuation and the SFR, does not completely cancel the residual slope that is still -0.13 . However, it is clear from the comparison of Figs. 8 and 10 that this remaining residual slope is much less significant (Spearman rank correlation coefficient of -0.36 instead of -0.5 to -0.7 ).

No estimation of the dust attenuation would be available in most cases where one has to use the [OII] or the $\mathrm{H} \beta$ emission 

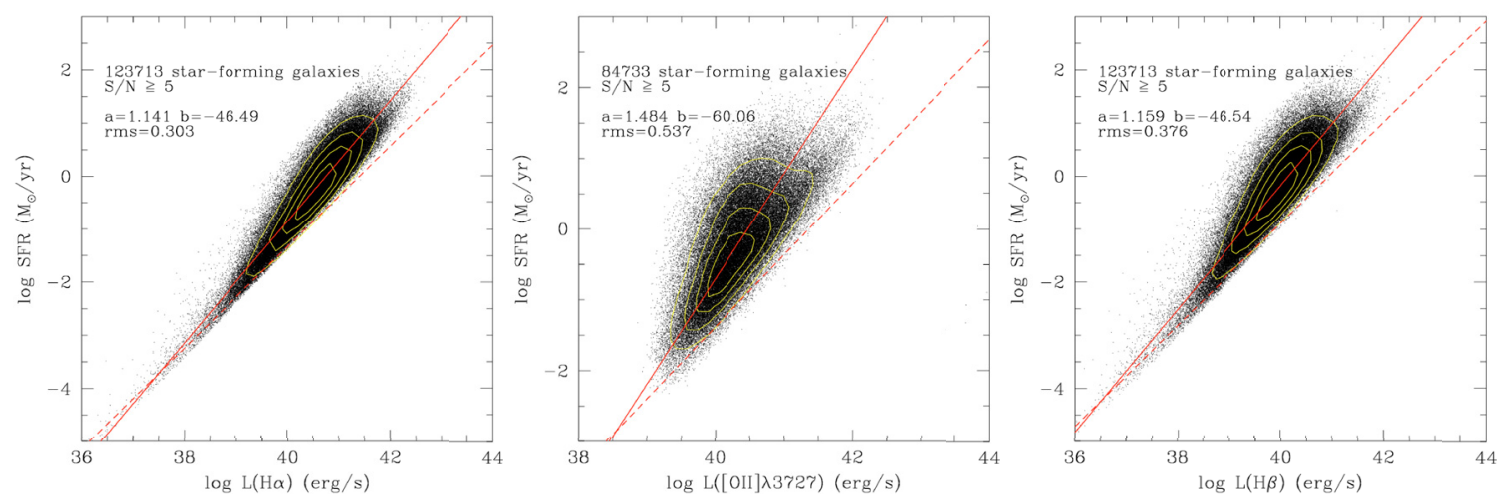

Fig. 11. Relation between the SFR (logarithm of $M_{\odot} / \mathrm{yr}$ ) and the $\mathrm{H} \alpha$ (left), [OII] (center), or $\mathrm{H} \beta$ (right) observed emission-line luminosities (logarithm of erg/s) for starforming galaxies in the SDSS DR4 data. The solid line is a least-square fit to the data (errors on $x$ and $y$ ). The dashed lines show the intrinsic calibrations obtained with dust estimated with the Balmer decrement method (see Fig. 3). Isodensity contours are overplotted in white.

line in order to compute an SFR. This is basically because the $\mathrm{H} \alpha$ line is commonly used to estimate the dust attenuation, and there is no reason to use another line if the H $\alpha$ line is measured.

Figure 10 (top-right) shows the results obtained when using the standard Kennicutt (1992) [OII $]^{\mathrm{i}}$ calibration on data uncorrected for dust attenuation. The dispersion is $0.48 \mathrm{dex}$ which is high. There is also a significant systematic shift of -0.83 dex, plus a significant (Spearman-rank correlation coefficient of -0.68 ) residual slope of -0.47 . Figure 11 (center) shows the relation between the observed [OII] emission line luminosity and the SFR in SDSS DR4 data. We obtain the following best-fit values:

$$
\left\{\begin{aligned}
\log \eta_{[\mathrm{OII}]} & =60.06 \pm 1.9 \\
\epsilon_{[\mathrm{OII}]} & =1.484 \pm 0.05
\end{aligned}\right.
$$

But, as we can see from Fig. 10 (middle-right), the dispersion ( $0.54 \mathrm{dex})$ is higher than the results obtained with an assumed mean correction (see Sect. 4.2, dispersion of $0.48 \mathrm{dex}$ ), and the correlation between observed [OII] luminosity and SFR is rather poor. Nevertheless, this new calibration has the advantage of showing a smaller residual slope of -0.22 , also much less significant (Spearman rank correlation coefficient of -0.25 ).

Figure 11 (right) shows the relation between the observed $\mathrm{H} \beta$ emission line luminosity and the SFR in SDSS DR4 data, which gives the following parameters:

$$
\left\{\begin{aligned}
\log \eta_{\mathrm{H} \beta} & =46.54 \pm 0.04 \\
\epsilon_{\mathrm{H} \beta} & =1.159 \pm 0.001
\end{aligned}\right.
$$

As shown in Fig. 10 (bottom), this new calibration shows slight improvement as compared to the method of an assumed mean correction: dispersion of 0.38 dex instead of $0.39 \mathrm{dex}$, smaller residual slope of -0.19 , smaller Spearman rank correlation coefficient of -0.41 for this residual slope.

The two-parameters calibrations presented in this section do not show significant residual slopes as compared to the method of the assumed mean correction. Unfortunately, the gain from having no more significant residual slopes is diminished by the still high dispersion. We claim that these new self-consistent single-line calibrations are, however, useful when one has no idea of the mean dust attenuation to assume. As stated before, the use of the wrong mean correction could instead lead to a non-negligible systematic bias.

\subsubsection{Discussion of previous works}

Another way to correct self-consistently for dust attenuation, as long as for other parameters (e.g. metallicity, ionization degree, etc.), is to use the absolute magnitude. Indeed, the absolute magnitude provides an estimate of the dust attenuation through the general correlation between mass and dust attenuation in galaxies. It also provides an estimate of the metallicity through the luminosity-metallicity relation. These calibrations are only derived for the [OII] line. Again, it is better to correct directly for dust attenuation using the Balmer decrement when $\mathrm{H} \alpha$ is observed.

The Moustakas et al. (2006) [OII] calibration is based on a correction with $B$-band, $k$-corrected absolute magnitude, and it now starts to be widely used in the literature. However, since the correction is provided by the authors in few discrete points (see Table 2 of their paper), it is not clear how to interpolate between them. The common approach now used in other works is to calculate a global linear regression. Figure 12 shows that this approach is not valid in the whole domain. We thus provide a parabolic fit that leads to the following final formula:

$\log \mathrm{SFR}=\log L([\mathrm{OII}])-41+6.86+0.862 \times M_{B}+0.027 \times M_{B}^{2},(20)$

where $M_{B}$ is the $B$-band $k$-corrected absolute magnitude in the Vega system $\left(B_{\text {Vega }}=B_{\mathrm{AB}}+0.09\right)$.

Figure 13 (left) shows how the results obtained with the Moustakas et al. (2006) calibration compares with the reference CL01 SFR. This calibration shows a significant systematic shift of -0.22 dex. However, the dispersion of 0.43 dex is better than previous calibrations from the observed [OII] luminosity. We observe also a non-negligible residual slope of -0.30 (Spearman rank correlation coefficient of -0.46), but not stronger than the residual slope obtained with the method of the assumed mean correction.

The Weiner et al. (2007) [OII] calibration is based on a correction with $H$-band, $k$-corrected absolute magnitude. The authors give the slope of the relation between the $\log \left([\mathrm{OII}]_{\mathrm{obs}} / \mathrm{H} \alpha^{i}\right)$ ratio and the $H$-band, $k$-corrected absolute magnitude: $+0.23 \mathrm{dex} / \mathrm{mag}$. The zero-point is obtained by applying the $0.68 \operatorname{dex} A(\mathrm{H} \alpha)$ extinction to the $\log \left([\mathrm{OII}]_{\mathrm{obs}} / \mathrm{H} \alpha_{\mathrm{obs}}\right)$ ratio of -0.32 dex at $M_{\mathrm{H}}=-21$. The final formula is

$\log \mathrm{SFR}=\epsilon_{\mathrm{H} \alpha}^{\mathrm{i}}\left(\log L([\mathrm{OII}])-0.23 \times M_{\mathrm{H}}-3.83\right)-\log \eta_{\mathrm{H} \alpha}^{\mathrm{i}},(21)$

where $M_{\mathrm{H}}$ is the $H$-band $k$-corrected absolute magnitude in the $\mathrm{AB}$ system. 


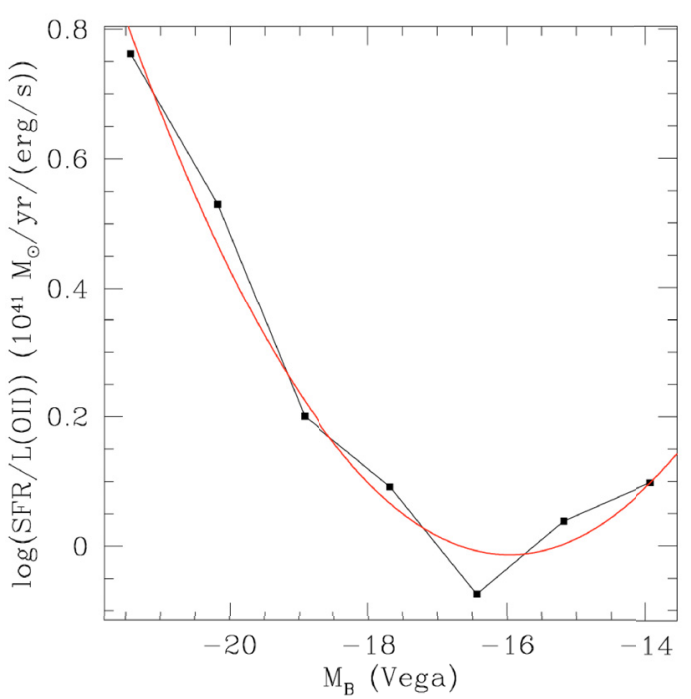

Fig. 12. Relation between the SFR efficiency factor of the [OII] emission line and the $B$-band $k$-corrected absolute magnitude (Vega system). The data points are the values given in Table 2 of Moustakas et al. (2006). The solid curve is our 2nd-degree polynomial least-square fit with the coefficients given in the text.
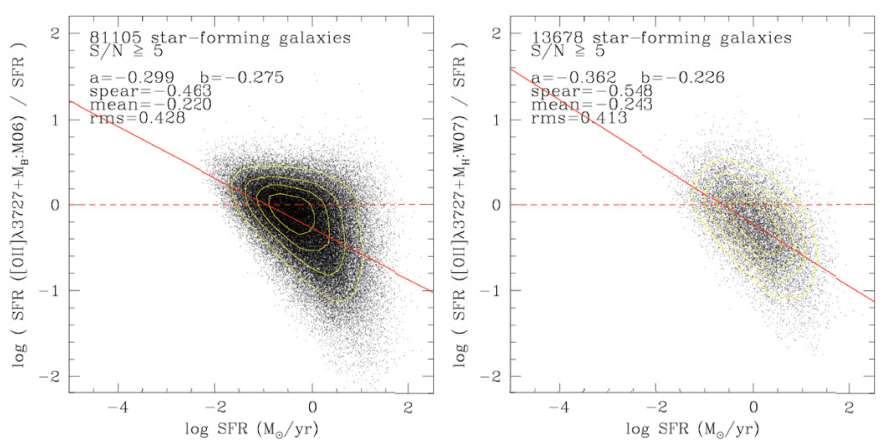

Fig. 13. Same legend as in Fig. 2. The calibrations are: left: Moustakas et al. (2006) $[\mathrm{OII}]+M_{B}$ calibration; right: Weiner et al. (2007) $[\mathrm{OII}]+M_{\mathrm{H}}$ calibration.

Figure 13 (right) shows how the results obtained with the Weiner et al. (2007) calibration compares with the reference CL01 SFR. The mean of the residuals is $-0.24 \mathrm{dex}$, the dispersion of 0.41 dex is better than the calibration on the observed [OII] luminosity, but it is still significantly high. Moreover, this smaller dispersion may only be due to the small number of galaxies (13678) with an available measurement of $M_{\mathrm{H}}$ in our sample. Finally, there is also a significant residual slope in this calibration of -0.36 (Spearman rank correlation coefficient of $-0.55)$.

We conclude that the calibrations proposed by Moustakas et al. (2006) and Weiner et al. (2007) lead to poor results with the SDSS DR4 data. They do neither significantly better nor significantly worse than the results obtained with the method of the assumed mean correction, or with our direct calibrations between the SFR and observed line luminosities.

\subsubsection{Two-line calibrations}

Finally we consider the general case where no estimation of the dust attenuation is available. Moustakas et al. (2006) and Weiner et al. (2007) have produced calibrations of the $[\mathrm{OII}] / \mathrm{H} \alpha^{\mathrm{i}}$ line ratio against $B$-band or $H$-band absolute magnitudes, respectively. Following our idea of using only emission-line measurements, we explored a possible correlation between $[\mathrm{OII}] / \mathrm{H} \alpha^{\mathrm{i}}$ (observed-to-intrinsic) and $[\mathrm{OII}] / \mathrm{H} \beta$ (observed-toobserved) line ratios. Figure 14 (left) shows the best-fit relation, as defined by the following equation:

$$
\log \left([\mathrm{OII}] / \mathrm{H} \alpha^{\mathrm{i}}\right)=3.243( \pm 0.3) \cdot \log ([\mathrm{OII}] / \mathrm{H} \beta)-1.74( \pm 0.09) \text {. }
$$

The mean and the rms of the residuals around the fitted line are respectively $-6 \times 10^{-10}$ dex and 0.37 dex. The objects plotted upward the dashed line in Fig. 14 (left) have negative dust attenuation when we derive them from $\mathrm{H} \alpha / \mathrm{H} \beta$ ratio with the assumption of a constant intrinsic Balmer ratio. Putting Eqs. (9) and (22) together, we obtain the following calibration formula:

$$
\log \mathrm{SFR}=\epsilon_{\mathrm{H} \alpha}^{\mathrm{i}}(\log L([\mathrm{OII}])-3.243 \cdot \log ([\mathrm{OII}] / \mathrm{H} \beta)+1.74) \log \eta_{\mathrm{H} \alpha}^{\mathrm{i}} \cdot(23
$$

Figure 14 (center) shows the quality of the recovered SFR as compared to the reference CL01 SFR. We see that our new calibration shows no significant systematic shift (0.02 dex) and no significant residual slope ( 0.01 with a Spearman-rank correlation coefficient of 0.06). Moreover, our new calibration shows a dispersion of 0.29 dex that, while being still higher than the results obtained with a correction for dust attenuation, is the best result obtained in Sect. 4 between all other possibilities: assumed mean dust correction, direct calibration with observed line luminosities, or self-consistent correction with the absolute magnitude.

We also explored the results obtained by doing a direct leastsquare fit of the SFR as a function of the [OII] line luminosity and the $[\mathrm{OII}] / \mathrm{H} \beta$ line ratio, without assuming the underlying $\mathrm{H} \alpha$ calibration. We find the following second-degree best-fit relation:

$$
\begin{array}{r}
\log \mathrm{SFR}=-39.21+0.982 \cdot \log L([\mathrm{OII}])-2.396 \cdot \log ([\mathrm{OII}] / \mathrm{H} \beta) \\
-0.3354 \cdot \log ([\mathrm{OII}] / \mathrm{H} \beta)^{2}
\end{array}
$$

Figure 14 (right) shows the quality of this other calibration as compared to the reference CL01 SFR. As compared to Eq. (23), the SFR derived with Eq. (24) show a zero systematic shift and a smaller dispersion of 0.23 dex. Nevertheless, this smaller dispersion is diminished by a residual slope of -0.12 (Spearman-rank correlation coefficient of -0.31).

Because it is much easier to use and it gives better results, our calibrations based on only two observed line fluxes ([OII] and $\mathrm{H} \beta$ ) should be preferred in most cases with no available correction for dust attenuation. The minimum uncertainty when dealing with data not corrected for dust attenuation is then only 0.23 dex. We discuss in the following section the different cases where it is better to use Eq. (23) or Eq. (24).

\subsection{The self-consistent correction on samples with different properties}

It is clear at this stage of our study that all the calibrations derived or studied in Sect. 4 (including those by Moustakas et al. 2006 and Weiner et al. 2007), which are based on observed quantities, depend on the properties of the reference sample. Nevertheless, we emphasize that this statistically significant sample such as the SDSS DR4 data, with a large selection function, is not expected to be strongly biased in favor of one particular population of galaxies.

It might be useful, however, to know how these calibrations are sensitive to the variations in the properties of the studied sample, especially in terms of dust or metallicity. 

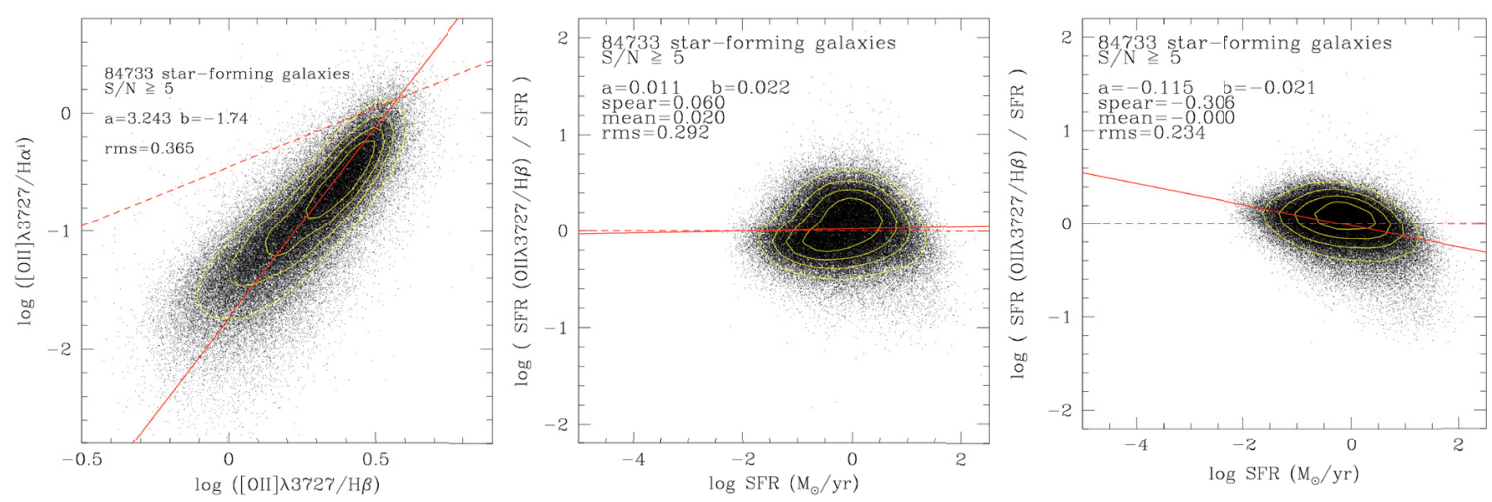

Fig. 14. Left: relation between the $[\mathrm{OII}] / \mathrm{H} \alpha^{\mathrm{i}}$ (observed-to-intrinsic) and the $[\mathrm{OII}] / \mathrm{H} \beta$ (observed-to-observed) line ratios for starforming galaxies in the SDSS DR4 data. The solid line is a least-square fit to the data (errors on $x$ and $y$ ). The dashed line is the $\mathrm{H} \alpha=2.85 \cdot \mathrm{H} \beta$ line. Isodensity contours are overplotted in white. Center and right: same legend as in Fig. 2. The calibrations are the new [OII]/H $\beta$ calibrations (center: Eq. (23), right: Eq. (24)).
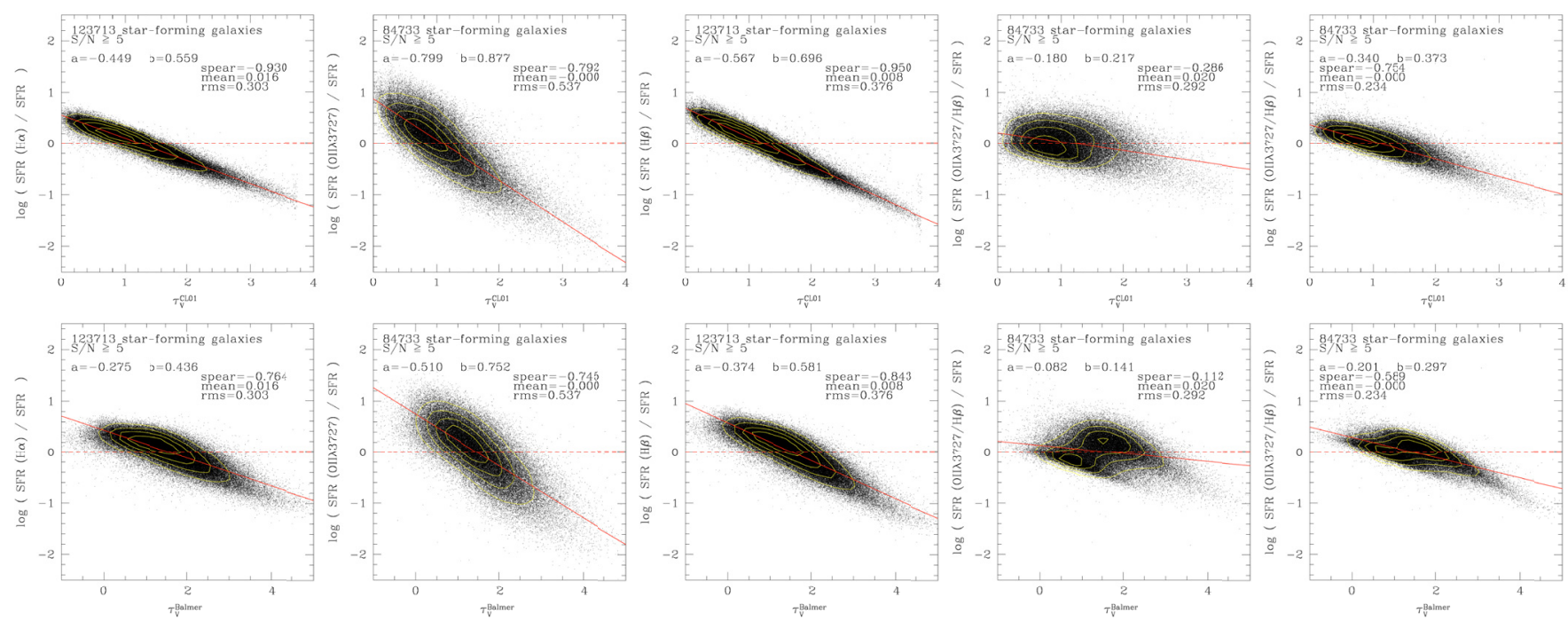

Fig. 15. Same legend as in Fig. 2, except that the residuals of the SFR calibration are now plotted as a function of the dust attenuation estimated with the CL01 method (top) or with the Balmer decrement method (bottom). The studied calibrations are, from left to right: $\mathrm{H} \alpha,[\mathrm{OII}], \mathrm{H} \beta,[\mathrm{OII}] / \mathrm{H} \beta$ (Eq. (23)), and [OII]/H $\beta$ (Eq. (24)). All calibrations are related to observed quantities.

\subsubsection{Dependence on dust}

We now study the residuals of our new calibrations in Fig. 15, as compared to the reference CL01 SFR, as a function of the dust attenuation estimated either with the CL01 method or with the assumption of a constant intrinsic Balmer ratio. This has been done for the poor quality single-line calibrations $(\mathrm{H} \alpha,[\mathrm{OII}]$, and $\mathrm{H} \beta$, see Fig. 10) and for the better quality [OII]/ $\mathrm{H} \beta$ calibration (see Fig. 14 center and right). In all cases, the means of the residuals are very low, which is expected as we always derived the best-fit calibration.

For the single-line calibrations, we obtained very significant correlations between the residuals and the dust attenuation, with Spearman-rank correlation coefficients on the order of -0.75 to -0.95 . Thus, we can derive linear relations that may be used to correct these calibrations for a desired study, for which one has a rough estimate of the mean dust attenuation. The corrective formula as derived from Fig. 15 is

$$
\log \left(\mathrm{SFR}^{\mathrm{corr}}\right)=\log (\mathrm{SFR})-a \times \tau_{V}-b
$$

The $a$ and $b$ coefficients are summarized in Table 1 . This correction formula give a zero correction for a dust attenuation equal
Table 1. Coefficients to use to correct the SFR derived from an observed calibration, as a function of the dust attenuation following Eq. (25).

\begin{tabular}{lrrrr}
\hline \hline Method & \multicolumn{2}{c}{ CL01 } & \multicolumn{2}{c}{ Balmer } \\
calibration & $a$ & $b$ & $a$ & $b$ \\
\hline $\mathrm{H} \alpha$ & -0.45 & 0.56 & -0.28 & 0.44 \\
{$[\mathrm{OII}]$} & -0.80 & 0.88 & -0.51 & 0.75 \\
$\mathrm{H} \beta$ & -0.57 & 0.70 & -0.37 & 0.58 \\
{$[\mathrm{OII}] / \mathrm{H} \beta$ (Eq. (23)) } & -0.18 & 0.22 & -0.082 & 0.14 \\
{$[\mathrm{OII}] / \mathrm{H} \beta$ (Eq. (24)) } & -0.34 & 0.37 & -0.20 & 0.30 \\
\hline
\end{tabular}

to the mean value in the SDSS DR4 sample: $\tau_{V}^{\mathrm{CL} 01}=1.21$ in the $\mathrm{H} \alpha$ and $\mathrm{H} \beta$ cases, and for $\tau_{V}^{\mathrm{CL} 01}=1.1$ in the [OII] case.

For the $[\mathrm{OII}] / \mathrm{H} \beta$ two-line calibration given in Eq. (23), we see that the residuals no longer show any strong significant correlation with the dust attenuation: smaller slopes, and smaller Spearman-rank correlation coefficients of -0.1 to -0.3 . This result shows that the $[\mathrm{OII}] / \mathrm{H} \beta$ calibration given in Eq. (23) is not significantly sensitive to variations in the dust attenuation. This calibration can thus reliably been used in any sample without applying a correction. This conclusion is not true for the $[\mathrm{OII}] / \mathrm{H} \beta$ 

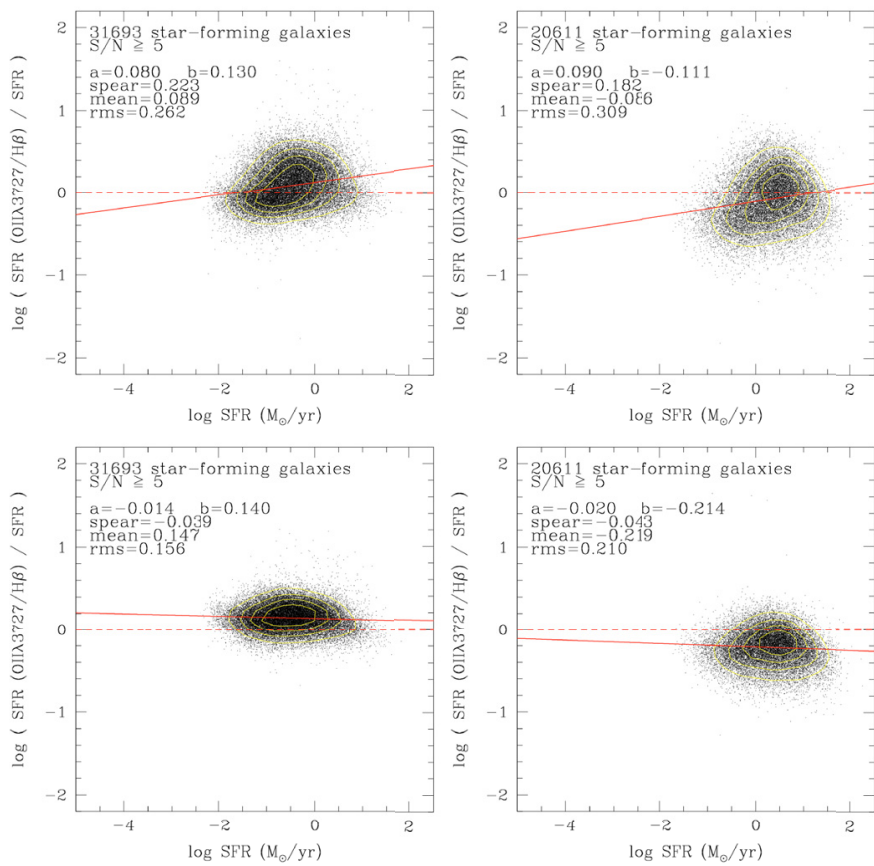

Fig. 16. Same legend as in Fig. 2. The calibration is the new [OII]/H $\beta$ calibration given by Eq. (23) (top) or Eq. (24) (bottom), applied to the two dust subsamples defined in Sect. 2. Left: $\left\langle\tau_{V}^{\mathrm{CL} 01}\right\rangle=0.63$, right: $\left\langle\tau_{V}^{\mathrm{CL} 01}\right\rangle=1.84$.

calibration given in Eq. (24), which shows similar, but lower, residual slopes as compared to single-line calibrations.

Figure 16 shows the $[\mathrm{OII}] / \mathrm{H} \beta$ calibrations applied on the two subsamples defined in Sect. 2, with two different dust properties: $\left\langle\tau_{V}^{\mathrm{CL} 01}\right\rangle=0.63$ and $\left\langle\tau_{V}^{\mathrm{CL} 01}\right\rangle=1.84$. It confirms that the calibration defined in Eq. (23) is reliable when applied to samples with different dust properties. The dispersion does not significantly changes, and the observed residual slopes are not significant (Spearman-rank correlation coefficients on the order of 0.2). In both cases, the observed systematic shifts agree with the correction formula given in Eq. (25) and the coefficients given in Table 1.

\subsubsection{Dependence on metallicity}

We also studied the residuals of our new calibrations, as compared to the reference CL01 SFR, as a function of the gas-phase oxygen abundance estimated with the CL01 method. For the single-line calibrations, Fig. 17 shows a significant correlation between these two quantities with Spearman-rank correlation coefficients on the order of -0.5 to -0.6 . Thus, we can derive linear relations, which may be used to correct these calibrations for a desired study, for which one has a rough estimate of the mean metallicity. The corrective formula as derived from Fig. 17 is

$\log \left(\mathrm{SFR}^{\mathrm{corr}}\right)=\log (\mathrm{SFR})-a \times x-b$

with $x=12+\log (\mathrm{O} / \mathrm{H})$. The $a$ and $b$ coefficients are summarized in Table 2 . This correction formula gives an approximately zero correction for a metallicity equal to the mean value in the SDSS DR4 sample: $12+\log (\mathrm{O} / \mathrm{H})=8.89$.

For both $[\mathrm{OII}] / \mathrm{H} \beta$ calibrations, we fitted a second-degree curve to the residual, which leads to the following corrective formula:

$\log \left(\mathrm{SFR}^{\mathrm{corr}}\right)=\log (\mathrm{SFR})-a \times x^{2}-b \times x-c$
Table 2. Coefficients to be used to correct the SFR derived from an observed calibration, as a function of the gas-phase oxygen abundance following Eq. (26). (first three calibrations) or Eq. (27) (two last calibrations).

\begin{tabular}{lrrr}
\hline \hline Calibration & $a$ & $b$ & $c$ \\
\hline $\mathrm{H} \alpha$ & -0.53 & 4.80 & \\
{$[\mathrm{OII}]$} & -1.50 & 13.35 & \\
$\mathrm{H} \beta$ & -0.74 & 6.67 & \\
{$[\mathrm{OII}] / \mathrm{H} \beta$ (Eq. (23)) } & 1.47 & -25.18 & 107.5 \\
{$[\mathrm{OII}] / \mathrm{H} \beta$ (Eq. (24)) } & 0.51 & -8.96 & 39.3 \\
\hline
\end{tabular}

with $x=12+\log (\mathrm{O} / \mathrm{H})$. The $a, b$, and $c$ coefficients are summarized in Table 2.

For the $[\mathrm{OII}] / \mathrm{H} \beta$ two-lines calibration given in Eq. (24), we see that the residuals no longer show any strong significant correlation with the metallicity, with a Spearman-rank correlation coefficients of 0.08 . This result shows that the $[\mathrm{OII}] / \mathrm{H} \beta$ calibration given in Eq. (24) is not significantly sensitive to variations in the metallicity. This conclusion is not true for the $[\mathrm{OII}] / \mathrm{H} \beta$ calibration given in Eq. (23), which shows a Spearman correlation coefficient of 0.57 .

Figure 18 shows the $[\mathrm{OII}] / \mathrm{H} \beta$ calibrations applied on the two subsamples defined in Sect. 2, with two different metallicity properties: $\langle 12+\log (\mathrm{O} / \mathrm{H})\rangle=8.68$ and $\langle 12+\log (\mathrm{O} / \mathrm{H})\rangle=$ 9.07. It confirms that the calibration defined in Eq. (24) is reliable when applied to samples with different dust properties: the dispersion does not significantly change. In both cases, the observed systematic shifts agree with the correction formula given in Eq. (27) and the coefficients given in Table 2.

We conclude that:

- the $[\mathrm{OII}] / \mathrm{H} \beta$ calibration given in Eq. (23) is weakly sensitive to variations in dust attenuation. It can be applied to samples with an assumed metallicity, using the corrective formula given in Eq. (27);

- the $[\mathrm{OII}] / \mathrm{H} \beta$ calibration given in Eq. (24) is not sensitive to variations in metallicity. It can be applied to samples with an assumed dust attenuation, using the corrective formula given in Eq. (25).

\section{Conclusions}

We draw the following conclusions from our study:

- as already shown in many previous studies, we confirm from SDSS DR4 data that the best emission-line calibration of the SFR is based on $\mathrm{H} \alpha^{\mathrm{i}}$, this one being corrected for dust attenuation (see Sect. 3.2.1). This calibration has an uncertainty of 0.17 dex;

- if the dust has been estimated from the wrong assumption of a constant intrinsic Balmer ratio, the standard oneparameter Kennicutt (1998) scaling law gives adequate results. Nevertheless, the SDSS DR4 data has shown a -0.11 dex systematic shift that can be corrected either by applying a mean shift in the opposite direction or by using a two-parameter power law (see Figs. 2, 3, and Eq. (7));

- when $\mathrm{H} \alpha^{\mathrm{i}}$ is not observed but a correction for dust attenuation is still available, the Kennicutt (1998) law based on $[\mathrm{OII}]^{\mathrm{i}}$ (and corrected for dust by Kewley et al. 2004) gives good results but shows a higher dispersion (see Fig. 2);

- this dispersion may be reduced by taking the dependence of $[\mathrm{OII}]^{\mathrm{i}}$ on the metallicity into account. However, the metallicity correction proposed by Kewley et al. (2004) does not 

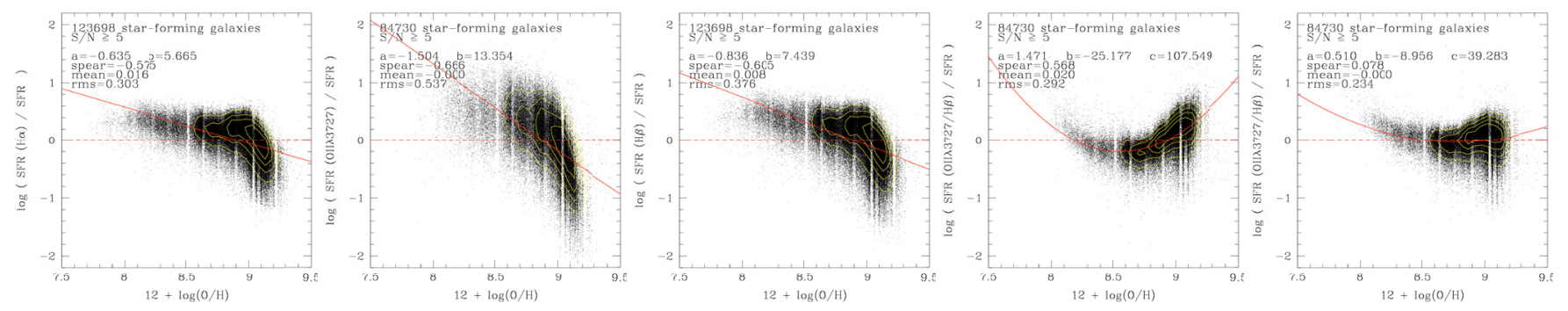

Fig. 17. Same legend as in Fig. 2, except that the residuals of the SFR calibration are now plotted as a function of the gas-phase oxygen abundance estimated with the CL01 method. The calibrations are, from left to right: $\mathrm{H} \alpha,[\mathrm{OII}], \mathrm{H} \beta,[\mathrm{OII}] / \mathrm{H} \beta$ (Eq. (23)), and [OII]/H $\beta$ (Eq. (24)). All calibrations are related to observed quantities.
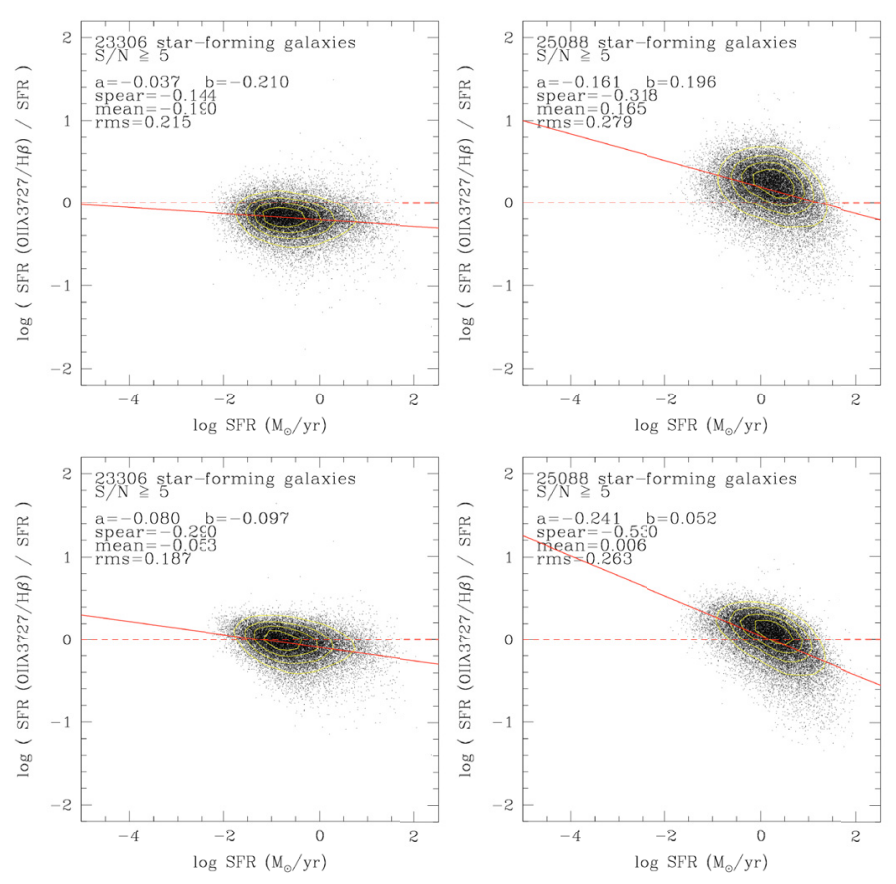

Fig. 18. Same legend as in Fig. 2. The calibration is the new $[\mathrm{OII}] / \mathrm{H} \beta$ calibration given by Eq. (23) (top) or Eq. (24) (bottom), applied to the two metallicity subsamples defined in Sect. 2. Left: $\langle 12+\log (\mathrm{O} / \mathrm{H})\rangle=$ 8.68 , right: $\langle 12+\log (\mathrm{O} / \mathrm{H})\rangle=9.07$.

lead to a significant improvement, while it relies on a very uncertain calibration of the metallicity, as shown by Kewley \& Ellison (2008) (see Figs. 4 and 5);

- a very good correction of this effect is instead obtained by using the $[\mathrm{OII}]^{\mathrm{i}}$ and $\mathrm{H} \beta^{\mathrm{i}}$ lines together or, even better, $\mathrm{H} \beta^{\mathrm{i}}$ alone (see Eqs. (9)-(11));

- we caution the reader against using the inadequate SFR calibration when data is corrected for dust estimated by another method (e.g. CL01 method). If the method of deriving dust attenuation is not biased towards the wrong assumption of a constant intrinsic Balmer ratio, then a different SFR calibration should be used (see Sect. 3.3.1, Fig. 6, Eqs. (12)-(14));

- we advise the reader not to use dust estimated from SEDfitting to calculate SFR from emission lines. This method leads to highly uncertain results because of the high dispersion in the stellar-to-gas attenuation ratio (see Fig. 7). We emphasize that dust attenuation estimated from SED-fitting would be reliable only if it is applied to the light emitted by stars, not to emission lines;

- when no estimation of the dust attenuation is available, it is common in the literature to assume a mean correction, typically $A_{V}=1$. We advise the reader to use this method with great care for two reasons: (i) the SFRs recovered with the assumption of a constant dust attenuation are biased by a non-negligible residual slope, coming from the correlation between dust attenuation and SFR (see Sect. 4.1.1 and Fig. 8); (ii) additional biases come from the choice of the assumed dust attenuation, which is likely not to be the right one. The choice of a wrong assumed dust attenuation leads to non-negligible systematic shifts (see Sect. 4.1.2);

- we derived new direct calibrations between the SFR and the observed line luminosities which are still quite poor in terms of dispersion, even if they no longer show a significant residual slope (see Figs. 10 and 11, Eqs. (17)-(19));

- the calibrations proposed by Moustakas et al. (2006) or Weiner et al. (2007), which include a correction based on the $B$-band or $H$-band, $k$-corrected absolute magnitudes, do neither significantly better nor significantly worse (see Fig. 13);

- we derived two new two-line calibrations based only on the [OII] and $\mathrm{H} \beta$ observed line flux. These calibrations give the best results among all calibrations based on observed quantities (i.e. not reliably corrected for dust attenuation). It shows no systematic shift, no significant residual slope, and a reduced dispersion (see Fig. 14, Eqs. (23) and (24)). The minimum uncertainty with data not corrected for dust attenuation is 0.23 dex;

- we studied the relation between the residuals of our calibrations based on observed quantities and the dust attenuation (see Fig. 15). There is a clear correlation for single-line calibrations, which can be corrected (see Eq. (25) and Table 1). We also found a correlation between the residuals of our calibrations based on observed quantities (see Fig. 17), which can be corrected for single-line calibrations using Eq. (26) and Table 2;

- among our two new $[\mathrm{OII}] / \mathrm{H} \beta$ calibrations, the one defined by Eq. (23) is designed to be used on a sample with an assumed metallicity and an unknown dust attenuation, while the one defined by Eq. (24) gives better results on samples with unknown metallicity and an assumed dust attenuation.

Acknowledgements. We warmly thank J. Brinchmann, G. Zamorani, S. Charlot, and T. Contini for valuable discussions concerning this work. We thank the anonymous referee for very useful comments that have significantly improved the paper. F. Lamareille thanks the Osservatorio Astronomico di Bologna and the COSMOS consortium for a post-doctoral fellowship. The physical properties of SDSS galaxies were produced by a collaboration of researchers (currently or formerly) at the MPA and the JHU. The team is made up of Stéphane Charlot, Guinevere Kauffmann, and Simon White (MPA), Tim Heckman (JHU), Christy Tremonti (University of Arizona - formerly JHU) and Jarle Brinchmann (Centro de Astrofísica da Universidade do Porto - formerly MPA). 


\section{References}

Adelman-McCarthy, J. K., Agüeros, M. A., Allam, S. S., et al. 2006, ApJS, 162, 38

Bicker, J., \& Fritze-v. Alvensleben, U. 2005, A\&A, 443, L19

Blanton, M. R., Schlegel, D. J., Strauss, M. A., et al. 2005, AJ, 129, 2562

Brinchmann, J., Charlot, S., White, S. D. M., et al. 2004, MNRAS, 351, 1151

Bruzual, G., \& Charlot, S. 2003, MNRAS, 344, 1000

Calzetti, D. 2001, PASP, 113, 1449

Chabrier, G. 2003, PASP, 115, 763

Charlot, S., \& Fall, S. M. 2000, ApJ, 539, 718

Charlot, S., \& Longhetti, M. 2001, MNRAS, 323, 887

Colless, M., Dalton, G., Maddox, S., et al. 2001, MNRAS, 328, 1039

Cortese, L., Boselli, A., Buat, V., et al. 2006, ApJ, 637, 242

Fioc, M., \& Rocca-Volmerange, B. 1997, A\&A, 326, 950

Kauffmann, G., Heckman, T. M., Tremonti, C., et al. 2003a, MNRAS, 346, 1055

Kauffmann, G., Heckman, T. M., White, S. D. M., et al. 2003b, MNRAS, 341, 33

Kennicutt, R. C. 1998, ARA\&A, 36, 189

Kennicutt, Jr., R. C. 1992, ApJ, 388, 310

Kewley, L. J., \& Dopita, M. A. 2002, ApJS, 142, 35

Kewley, L. J., \& Ellison, S. L. 2008, ApJ, 681, 1183

Kewley, L. J., Geller, M. J., \& Jansen, R. A. 2004, AJ, 127, 2002

Kroupa, P. 2001, MNRAS, 322, 231
Lamareille, F., Mouhcine, M., Contini, T., Lewis, I., \& Maddox, S. 2004, MNRAS, 350, 396

Lamareille, F., Contini, T., Brinchmann, J., et al. 2006, A\&A, 448, 907

Lamareille, F., Brinchmann, J., Contini, T., et al. 2008, A\&A, 495, 53

Le Fèvre, O., Vettolani, G., Garilli, B., et al. 2005, A\&A, 439, 845

Lequeux, J., Peimbert, M., Rayo, J. F., Serrano, A., \& Torres-Peimbert, S. 1979, A\&A, 80, 155

McGaugh, S. S. 1991, ApJ, 380, 140

Mouhcine, M., Lewis, I., Jones, B., et al. 2005, MNRAS, 362, 1143

Moustakas, J., Kennicutt, Jr., R. C., \& Tremonti, C. A. 2006, ApJ, 642, 775

Osterbrock, D. E. 1989, Astrophysics of gaseous nebulae and active galactic nuclei (Mill Valley, CA, University Science Books), 422

Pettini, M., \& Pagel, B. E. J. 2004, MNRAS, 348, L59

Richer, M. G., \& McCall, M. L. 1995, ApJ, 445, 642

Salpeter, E. E. 1955, ApJ, 121, 161

Seaton, M. J. 1979, MNRAS, 187, 73P

Skillman, E. D., Kennicutt, R. C., \& Hodge, P. W. 1989, ApJ, 347, 875

Spergel, D. N., Verde, L., Peiris, H. V., et al. 2003, ApJS, 148, 175

Tremonti, C. A., Heckman, T. M., Kauffmann, G., et al. 2004, ApJ, 613, 898

van Zee, L., Salzer, J. J., Haynes, M. P., O’Donoghue, A. A., \& Balonek, T. J. 1998, AJ, 116, 2805

Weiner, B. J., Papovich, C., Bundy, K., et al. 2007, ApJ, 660, L39

York, D. G., Adelman, J., Anderson, Jr., J. E., et al. 2000, AJ, 120, 1579 